\title{
SOLUTION OF THE INVERSE PROBLEM OF THE CALCULUS OF VARIATIONS
}

\author{
BY \\ JESSE DOUGLAS \\ PART I. INTRODUCTION
}

1. Formulation and background. The problem indicated in the title is one of the most important hitherto unsolved problems of the calculus of variations, namely:

Given any family of $\infty^{2 n}$ curves (paths) in $(n+1)$-dimensional space $\left(x, y_{j}\right)$, $(j=1, \cdots, n)$, as represented by a system of differential equations

$$
y_{i}^{\prime \prime}=F_{i}\left(x, y_{i}, y_{j}^{\prime}\right)
$$

$(i=1, \cdots, n)$;

to determine whether these curves can be identified with the totality of extremals of some variation problem

$$
\int \phi\left(x, y_{j}, y_{j}^{\prime}\right) d x=\min ,
$$

and in the affirmative case to find all the corresponding functions $\phi$.

The present paper solves this problem for the most important and interesting case of 3-dimensional space $(n=2)$, where the given family consists of $\infty^{4}$ curves defined by differential equations of the form

$$
y^{\prime \prime}=F\left(x, y, z, y^{\prime}, z^{\prime}\right), \quad z^{\prime \prime}=G\left(x, y, z, y^{\prime}, z^{\prime}\right),
$$

and the variation problem sought for is of the form

$$
\int \phi\left(x, y, z, y^{\prime}, z^{\prime}\right) d x=\min .
$$

Our essential results and methods have already been published in two preliminary notes $\left({ }^{1}\right)$.

Basically, our procedure consists in an application of the Riquier theory of systems of partial differential equations to a certain linear differential system $\subseteq$ on which the inverse problem can be made to depend. This differential system has already appeared-derived in a different way-in the interesting work, of little more than a decade ago, by D. R. Davis on the inverse problem $\left({ }^{2}\right)$; but, as he stated, its general solution-even existence-theoreticallypresented difficulties which he could not overcome.

Presented to the Society, January 1, 1941; received by the editors March 13, 1940.

(1) Numbers 8 and 9 of the list of references at the end of $\$ 2$.

(2) See numbers 6 and 7 of the list of references at the end of $\S 2$. 
In this paper we succeed in obtaining a complete solution of the differential system $\subseteq$ in the following sense. We determine in all cases whether or not a solution exists corresponding to given $(F, G)$-that is, a classification is made of all curve families $(F, G)$ into "extremal" and "nonextremal." In each case of consistency of the system $\subseteq$ we derive an equivalent completely integrable differential system [ऽ] which determines $\phi$. This system [ऽ] is either given explicitly - that is, the coefficients in its equations (all linear) are expressed as functions of the partial derivatives of $(F, G)$-or else (when this seems to lead to calculations too lengthy to be worth while) we describe precisely those differentiations and algebraic processes (additions and multiplications), finite in number, which will furnish this completely integrable system. The explicit formulation of [ऽ] is found to be feasible in all the important cases. Standard criteria of the theory of differential systems then enable us to determine the degree of generality of the solution $\phi$, that is, the number and nature of the arbitrary functions or constants which are involved.

It may be emphasized that the coordinates $\left(x, y_{j}\right)$ are by no means necessarily cartesian, but may be perfectly general point coordinates. Indeed, as is evident $a$ priori, the fundamental group of our problem is that of all (analytic) point transformations (or, otherwise interpreted, all coordinate transformations); for if the curves (1.1) are the extremals of the integral (1.2), this relation obviously continues to subsist, after arbitrary transformation of the variables $\left(x, y_{j}\right)$, between the transformed curves and the transformed integral.

We assume the functions $F_{i}, \phi$ and any others that may enter into our investigations to be analytic about a particular linear element $\left(x^{(0)}, y_{j}^{(0)}, y_{j}^{\prime(0)}\right)$ which we may take to be located at the origin along the $x$-axis, that is, with all coordinates zero.

The principal formulas of a tensor form of treatment of the inverse problem, based on parametric representation, have been given in one of our preliminary notes $\left({ }^{3}\right)$. In the present exposition the non-parametric form of representation, as in all the preceding formulas, will be employed throughout.

A nalytic formulation. Analytically expressed, our inverse problem consists in the solution for $\phi$, as unknown function, of the Euler-Lagrange equations

$$
\frac{\partial \phi}{\partial y_{i}}-\frac{d}{d x} \frac{\partial \phi}{\partial y_{i}^{\prime}}=0 \quad(i=1, \cdots, n) .
$$

Here the operator $d / d x$ denotes total differentiation with respect to $x$ along an arbitrary curve of the given family; that is, as applied to any function of $x, y_{i}, y_{j}^{\prime}$,

$$
\frac{d}{d x} \equiv \frac{\partial}{\partial x}+y_{j}^{\prime} \frac{\partial}{\partial y_{j}}+F_{j} \frac{\partial}{\partial y_{j}^{\prime}}
$$

(3) Number 8 of the list at the end of $\$ 2$. 
where, as throughout this paper, the summation convention applies to repeated indices.

In expanded form, the Euler-Lagrange equations are, then:

$$
\frac{\partial \phi}{\partial y_{i}}-\left(\frac{\partial}{\partial x}+y_{j}^{\prime} \frac{\partial}{\partial y_{j}}+F_{j} \frac{\partial}{\partial y_{j}^{\prime}}\right) \frac{\partial \phi}{\partial y_{i}^{\prime}}=0 \quad(i=1, \cdots, n),
$$

where $F_{j}$ are given and $\phi$ is to be found. Further, these equations must be solvable uniquely as a linear algebraic system for the quantities $F_{j}$ or $y_{j}^{\prime \prime}$, in order that the differential equations of the given curve family may have the prescribed form (1.1). Therefore a solution of (1.7) is required such that

$$
\operatorname{Det}\left|\phi_{i j}\right| \neq 0 \text {, }
$$

where

$$
\phi_{i j} \equiv \frac{\partial^{2} \phi}{\partial y_{i}^{\prime} \partial y_{i}^{\prime}} .
$$

The determinant (1.8) is, of course, the Hessian of $\phi$.

For $n=1$, or a 2-dimensional containing space, the solution of the inverse problem is classic, being given in the standard textbooks $\left({ }^{4}\right)$. The system (1.7) then consists of but a single equation, whose solvability for $\phi$ is assured by known existence theorems. Thus, any given family of $\infty^{2}$ curves in the plane, as defined by a differential equation

$$
y^{\prime \prime}=F\left(x, y, y^{\prime}\right),
$$

can be regarded as the totality of extremals of a problem

$$
\int \phi\left(x, y, y^{\prime}\right) d x=\min .
$$

Indeed, $\phi$ can be explicitly determined by quadratures if the differential equation (1.10) has been integrated: $y=f(x, a, b)$. The formula for $\phi$ involves essentially one arbitrary function of two arguments $\left({ }^{5}\right)$, so that the class of integrals corresponding to a given curve family assigned as extremals is quite extensive.

The difficulty of the problem resides in the case $n \geqq 2$, that is, for a 3 - or higher dimensional space. Then the number of equations (1.7) to be satisfied exceeds the number (one) of unknown functions, and for this reason it has always been presumed (correctly) that for arbitrarily given $F_{j}$ the equations (1.7) have no solution except the trivial one of any total derivative:

(4) See Bolza, Lectures on the Calculus of Variations, New York, 1931, pp. 31-32. Cf. also the reference to the work of Darboux in \$2.

(5) In this count we omit the arbitrary function $\nu(x, y)$ in the exact differential $d \nu(x, y)$ that may be added to $\phi\left(x, y, y^{\prime}\right) d x$. 


$$
\phi=\frac{d}{d x} \nu\left(x, y_{j}\right)=\frac{\partial \nu}{\partial x}+\frac{\partial \nu}{\partial y_{j}} y_{j}^{\prime},
$$

which evidently does not satisfy the condition (1.8) since each element (1.9) is equal to zero. In other words, the presumption is that a given family (1.1) of $\infty^{2 n}$ curves in $(n+1)$-space is, in general, not an extremal family.

However, it may be an indication of the difficulty of discussing the system (1.7) directly that hitherto no proof has ever been given of this surmisal. That is to say, no example has ever been given of a $2 n$-parameter curve family in $(n+1)$-space which is certainly not an extremal family $\left.{ }^{6}\right)$.

In the course of the general solution of the inverse problem that occupies this paper, many examples of nonextremal families are found and proved to be such; for instance, in $x y z$-space

$$
y^{\prime \prime}=y^{2}+z^{2}, \quad z^{\prime \prime}=y
$$

is a non-extremal family $\left({ }^{7}\right)$, so is

$$
y^{\prime \prime}=y^{2}+z^{2}, \quad z^{\prime \prime}=0\left({ }^{8}\right) ;
$$

and as many others as are desired can be constructed.

2. Historical survey. Darboux in his geometrical treatise $\left(^{9}\right)$ stated and solved the inverse problem for the 2-dimensional case.

G. Hamel, in 1903, gave a solution of a particular case of the inverse problem in 3-space where the assigned extremals are the totality of straight lines.

Along another line, Jacobi, in his fundamental memoir of 1837 on the calculus of variations, proved that if $\delta E=0$ is the variational equation for the Euler equation (of $2 n$th order):

$$
E \equiv \frac{\partial \phi}{\partial y}-\frac{d}{d x} \frac{\partial \phi}{\partial y^{\prime}}+\frac{d^{2}}{d x^{2}} \frac{\partial \phi}{\partial y^{\prime \prime}}-\cdots+(-1)^{n} \frac{d^{n}}{d x^{n}} \frac{\partial \phi}{\partial y^{(n)}}=0
$$

corresponding to a 2-dimensional problem of any order $n$

$$
\int \phi\left(x, y, y^{\prime}, y^{\prime \prime}, \cdots, y^{(n)}\right) d x=\min ,
$$

then $\delta E$ is always self-adjoint as a linear differential expression in $\delta y$.

In 1897, A. Hirsch proved that, conversely, if

$$
\omega\left(x, y, y^{\prime}, \cdots, y^{(2 n)}\right)
$$

is any differential expression whose variation $\delta \omega$ (a linear differential expres-

(6) Except in the preliminary notes to this paper, cited in Footnote 1.

(7) Cf. $\$ 18$.

(8) Cf. $\$ 17$.

( ${ }^{9}$ See, throughout, the list of references at the end of this section. 
sion in $\delta y$ ) is self-adjoint, then $\omega$ must be identifiable with the Euler expression $E$ corresponding to some integrand $\phi$, as defined by (2.1). Later, J. Kürschak extended this result to expressions involving partial derivatives.

D. R. Davis, in a Chicago dissertation of about a decade ago, proved the corresponding converse theorem for a system of $n$ differential expressions $\omega_{i}\left(x, y_{j}, y_{j}^{\prime}, y_{j}^{\prime \prime}\right)$ : if their variations $\delta \omega_{i}$ form a self-adjoint linear differential system in $\delta y_{j}$, then $\phi\left(x, y_{j}, y_{j}^{\prime}\right)$ exists such that the $\omega_{i}$ are the first members of Euler's equations for $\int \phi d x=\min$. On this basis, Davis transformed the inverse problem in $(n+1)$-space into the determination of multipliers $P_{i j}\left(x, y_{k}, y_{k}^{\prime}\right)$ for the given differential equations (1.1) such that the following differential expressions :

$$
E_{i}=P_{i j}\left\{F_{j}\left(x, y_{k}, y_{k}^{\prime}\right)-y_{j}^{\prime \prime}\right\},
$$

have variations $\delta E_{i}$ which are self-adjoint.

By imposing this condition of self-adjointness, Davis obtained for the multipliers $P_{i j}$ precisely the differential system $\subseteq$ which we shall derive in an entirely different way in the present paper (Part II) $\left({ }^{10}\right)$. In attempting, however, to solve the system $\subseteq$ even existence-theoretically, Davis met with difficulties which, as he stated, he was unable to overcome. He therefore contented him self with the study of certain three particular examples $\left({ }^{11}\right)$.

The present paper accomplishes the solution of the system $\subseteq$ from the existence-theoretic standpoint. This includes the determination in all the various cases of the existence and generality (in terms of arbitrary functions and constants) of the solution. In the case of existence of a solution, a completely integrable differential system is given, either explicitly or implicitly, for the determination of this solution.

Incidentally, we derive the fundamental differential system $\subseteq$ from an entirely new point of view, based on certain identities which we have found to be obeyed by the Euler expressions. Self-adjointness thus plays no rôle whatever in our theory and may be omitted entirely from consideration.

\section{REFERENCES}

1. G. Darboux, Leçons sur la Théorie Générale des Surfaces, Paris, 1894, §\$604, 605.

2. G. Hamel, Über die Geometrieen in denen die Geraden die Kürzesten sind, Mathematische Annalen, vol. 57 (1903), pp. 231-264.

${ }^{(10)}$ It should be remarked that Davis does not explicitly include in the system $\subseteq$ the important inequation $L N-M^{2} \neq 0$ (see formula (7.5)), which plays a very prominent role in our treatment of the system.

(i1) These are: (i) the straight lines, (ii) all circles orthogonal to a fixed plane, (iii) catenaries (cf. the example at the end of $\$ 12$ of the present paper, where this case is treated by a different method). Davis does not seem to have noticed explicitly that (ii) is equivalent to (i) by a point transformation, so that these examples are essentially identical from the standpoint of our problem (cf. the fifth paragraph in $\$ 1$ ). 
3. C. G. J. Jacobi, Zur Theorie der Variationsrechnung und der Differentialgleichungen, Werke, vol. 4.

4. A. Hirsch, Über eine charakteristische Eigenschaft der Differentialgleichungen der Variationsrechnung, Mathematische Annalen, vol. 49 (1897), pp. 49-72.

5. J. Kürschak, Über eine charakteristische Eigenschaft der Differentialgleichungen der Variationsrechnung, Mathematische Annalen, vol. 60 (1905), pp. 157-165.

6. D. R. Davis, The inverse problem of the calculus of variations in higher space, these Transactions, vol. 30 (1928), pp. 710-736.

7. D. R. Davis, The inverse problem of the calculus of variations in a space of $(n+1)$ dimensions, Bulletin of the American Mathematical Society, vol. 35 (1929), pp. 371-380.

8. J. Douglas, Solution of the inverse problem of the calculus of variations, Proceedings of the National Academy of Sciences, vol. 25 (1939), pp. 631-637.

9. J. Douglas, Theorems in the inverse problem of the calculus of variations, ibid., vol. 26 (1940), pp. 215-221.

The last two are preliminary notes to the present paper.

3. Statement of results. Because of the number and variety of our results, it seems desirable to present them first before proceeding to their detailed development and proof. In this section we therefore collect the theorems whose establishment is the goal of the main body, Part IV, of this paper. We shall also give an example of each of the important types which arise in our classification.

Of central importance is a certain matrix

$$
\Delta \equiv\left\|\begin{array}{lll}
A & B & C \\
A_{1} & B_{1} & C_{1} \\
A_{2} & B_{2} & C_{2}
\end{array}\right\|
$$

whose elements are known functions depending on the given curve family $(F, G)$, being expressions in the partial derivatives of $F, G$. Explicitly:

$$
\begin{aligned}
& A=\frac{d}{d x} F_{z^{\prime}}-2 F_{z}-\frac{1}{2} F_{z^{\prime}}\left(F_{y^{\prime}}+G_{z^{\prime}}\right), \\
& B=-\frac{d}{d x} F_{y^{\prime}}+\frac{d}{d x} G_{z^{\prime}}+2\left(F_{y}-G_{z}\right)+\frac{1}{2}\left(F_{y^{\prime}}-G_{z^{\prime}}\right)\left(F_{y^{\prime}}+G_{z^{\prime}}\right), \\
& C=-\frac{d}{d x} G_{y^{\prime}}+2 G_{y}+\frac{1}{2} G_{y^{\prime}}\left(F_{y^{\prime}}+G_{z^{\prime}}\right),
\end{aligned}
$$

where for the 3-dimensional case given in (1.3), the differential operator $d / d x$ of (1.6) is

$$
\frac{d}{d x} \equiv \frac{\partial}{\partial x}+y^{\prime} \frac{\partial}{\partial y}+z^{\prime} \frac{\partial}{\partial z}+F \frac{\partial}{\partial y^{\prime}}+G \frac{\partial}{\partial z^{\prime}} .
$$

$A_{1}, B_{1}, C_{1}$ are derived from $A, B, C$ by the formulas 


$$
\begin{aligned}
& A_{1}=\frac{d A}{d x}-F_{y^{\prime}} A-\frac{1}{2} F_{z^{\prime}} B, \\
& B_{1}=\frac{d B}{d x}-G_{y^{\prime}} A-\frac{1}{2}\left(F_{y^{\prime}}+G_{z^{\prime}}\right) B-F_{z^{\prime}} C, \\
& C_{1}=\frac{d C}{d x}-\frac{1}{2} G_{y^{\prime}} B-G_{z^{\prime}} C,
\end{aligned}
$$

while $A_{2}, B_{2}, C_{2}$ are derived from $A_{1}, B_{1}, C_{1}$ by the same formulas (recursion).

The rank of $\Delta$ is the principal basis of classification of curve families $(F, G)$ with respect to our inverse problem; accordingly, we begin by a separation into cases according to this rank, which will be followed by the appropriate sub-classifications in the statement of our theorems.

Case I. $\|A B C\|=0$, that is, $A=0, B=0, C=0$.

Case II.

$$
\left\|\begin{array}{lll}
A & B & C \\
A_{1} & B_{1} & C_{1}
\end{array}\right\|=0, \quad\|A B C\| \neq 0 .
$$

Case III.

$$
\left|\begin{array}{lll}
A & B & C \\
A_{1} & B_{1} & C_{1} \\
A_{2} & B_{2} & C_{2}
\end{array}\right|=0, \quad \| \begin{array}{ccc}
A & B & C \\
A_{1} & B_{1} & C_{1}
\end{array} \mid \neq 0 .
$$

Case IV.

$$
\left|\begin{array}{lll}
A & B & C \\
A_{1} & B_{1} & C_{1} \\
A_{2} & B_{2} & C_{2}
\end{array}\right| \neq 0
$$

Here, in writing a matrix $=0$, we mean that each determinant resulting therefrom by the suppression of columns (only) is equal to zero, and $\neq 0$ means that at least one such determinant is not equal to zero.

It is seen by reference to the recursion formulas (3.4) that the cases thus described are precisely those of rank $0,12,3$ of the matrix $\Delta$, respectively.

Case II is the most difficult and varied in its results. Fundamental in its treatment is the quadratic equation

$$
A \xi^{2}+B \xi+C=0,
$$

whose roots we denote by $\lambda, \mu$. It is important to distinguish the case of unequal roots from that of equal roots:

Case IIa. $B^{2}-4 A C \neq 0, \lambda \neq \mu$;

Case IIb. $B^{2}-4 A C=0, \lambda=\mu$. 
$\lambda, \mu$ are known functions of $x, y, z, y^{\prime}, z^{\prime}$ when the curve family is given, being algebraic functions of the partial derivatives of $F, G$.

The investigation of Case IIa leads to an interesting division into three subcases according to the following criteria:

Case IIa1. $\lambda \lambda_{z^{\prime}}-\lambda_{y^{\prime}}=0, \mu \mu_{z^{\prime}}-\mu_{y^{\prime}}=0$;

Case IIa2. $\lambda \lambda_{z^{\prime}}-\lambda_{y^{\prime}}=0, \mu \mu_{z^{\prime}}-\mu_{y^{\prime}} \neq 0$;

Case IIa3. $\lambda \lambda_{z^{\prime}}-\lambda_{y^{\prime}} \neq 0, \mu \mu_{z^{\prime}}-\mu_{y^{\prime}} \neq 0$.

For reasons that will appear in our later detailed discussion (cf. end \$10), we shall refer to these cases respectively as "separated," "semi-separated," and "non-separated."

Case IIb will be subdivided into

Case IIb1. $\lambda \lambda_{z^{\prime}}-\lambda_{y^{\prime}}=0$;

Case IIb2. $\lambda \lambda_{z^{\prime}}-\lambda_{y^{\prime}} \neq 0$.

Case IIb1 will be further divided into

Case IIb1'. $\lambda \lambda_{z^{\prime}}-\lambda_{y^{\prime}}=0,\left(\mathrm{IX}^{\prime}\right)=0$;

Case IIb1". $\lambda \lambda_{z^{\prime}}-\lambda_{y^{\prime}}=0,\left(\mathrm{IX}^{\prime}\right) \neq 0$ :

where $\left(\mathrm{IX}^{\prime}\right)$ is a certain expression in the partial derivatives of $F, G$, given explicitly by formula (14.10) modified according to the remarks following formula (15.2).

In Case III, let $\Delta_{1}, \Delta_{2}, \Delta_{3}$ denote the second order determinants contained in the matrix of $A, B, C$ and $A_{1}, B_{1}, C_{1}$ :

$$
\Delta_{1}=B C_{1}-B_{1} C, \quad \Delta_{2}=C A_{1}-C_{1} A, \quad \Delta_{3}=A B_{1}-A_{1} B .
$$

The vanishing or nonvanishing of

$$
D=\Delta_{1} \Delta_{3}-\Delta_{2}^{2}
$$

is important in the discussion of Case III ; accordingly, we subdivide this case:

Case IIIa. $D \neq 0$,

Case IIIb. $D=0$.

We shall indicate the generality of the solution of a given differential system by the notation

$$
\infty m_{1} f\left(n_{1}\right)+m_{2} f\left(n_{2}\right)+\cdots+m_{k} f\left(n_{k}\right)+p(12)
$$

to represent the presence in the general solution of $m_{1}$ arbitrary functions of $n_{1}$ arguments, $m_{2}$ arbitrary functions of $n_{2}$ arguments, $\cdots, m_{k}$ arbitrary functions of $n_{k}$ arguments, and $p$ arbitrary constants.

We remark that the extremals of $\int \phi d x=\min$ and $\int[\phi d x+d \nu(x, y, z)]=\min$, where $\nu$ denotes an arbitrary function, are evidently the same. For

$$
\int_{P_{1}}^{P_{2}}[\phi d x+d \nu(x, y, z)]=\int_{P_{1}}^{P_{2}} \phi d x+\nu\left(x_{2}, y_{2}, z_{2}\right)-\nu\left(x_{1}, y_{1}, z_{1}\right),
$$

(12) This symbol is due to E. Kasner, appearing in his review of Riquier's treatise on differential systems, Bulletin of the American Mathematical Society, vol. 19 (1913), p. 14. 
where $P_{1}\left(x_{1}, y_{1}, z_{1}\right)$ and $P_{2}\left(x_{2}, y_{2}, z_{2}\right)$ are any two points of space. Since the difference between the values of the two integrals is independent of the path followed from $P_{1}$ to $P_{2}$, a minimizing path for one is also minimizing for the other.

It is also evident that $\phi$ and $c \phi$, where $c$ denotes an arbitrary nonzero constant factor, have the same extremals.

In estimating the generality of the integrand $\phi\left(x, y, x, y^{\prime}, z^{\prime}\right)$ corresponding to given $F, G$, we shall omit the arbitrary function $\nu(x, y, z)$ and the arbitrary constant $c$, considering the class of integrands

$$
c \phi\left(x, y, z, y^{\prime}, z^{\prime}\right)+\frac{d}{d x} \nu(x, y, z)
$$

as defining essentially a single variation problem equivalent to $\int \phi d x=\min$.

We are now ready to state our results and to cite our illustrative examples. As has been said, in all cases where the given curve family is of extremal type, that is, a corresponding variation problem exists, we shall give (in Part IV) a completely integrable differential system for the determination of the integrand $\phi$. All these systems are linear in the partial derivatives of the unknown functions.

Theorem I. In Case I the given curve family is always of extremal type, and the generality of the corresponding variation problem is expressed by the symbol $\infty^{2 f(3)+2 f(2)}$.

This theorem is illustrated by the straight lines: $y^{\prime \prime}=0, z^{\prime \prime}=0$; also more generally by $y^{\prime \prime}=f\left(z^{\prime}\right), z^{\prime \prime}=0$, the function $f$ being arbitrary.

Theorem II. In Case IIa1 (separated) the given curve family is of extremal type and the generality of the corresponding variation problem is $\infty^{2 f(2)}$. The explicit determination of the integrand $\phi$ depends on the solution of two separate complete systems of linear partial differential equations of the first order $\left({ }^{13}\right)$, with respective unknown functions $\rho\left(x, y, z, y^{\prime}, z^{\prime}\right), \sigma\left(x, y, z, y^{\prime}, z^{\prime}\right)$; there are three equations in each system.

Example. $y^{\prime \prime}=F\left(x, y, y^{\prime}\right), z^{\prime \prime}=G\left(x, z, z^{\prime}\right)$, with $B \neq 0$. The inverse problem here separates into one in the $x y$-plane and one in the $x z$-plane, whose solutions by addition give the in tegrand $\phi$ for the space problem.

TheOREM III. In Case IIa2 (semi-separated) the solution of the inverse problem depends first on the solution for $\sigma$ of a certain system of linear partial differential equations of first order in that unknown function, which system may be rendered complete in the usual way by the adjunction of alternants. Its solution is then substituted in a system of three linear partial differential equations of first

(13) See the reference in Footnote 24. 
order involving $\rho$ together with $\sigma$, whereupon this system becomes a complete one for $\rho$. According to the number of linearly independent equations in the complete system for $\sigma(4,5$, or 6$)$, the generality of the integrand $\phi$ is $\infty^{1 f(2)+1 f(1)}$, or $\infty^{1 f(2)}$, or else $\phi$ is nonexistent, that is, the given curve family is nonextremal.

Example. The $\infty^{4}$ catenaries which lie in planes perpendicular to the $x z-$ plane (pictured as horizontal) and the directrix of each of which coincides with the trace of its plane upon the $x z$-plane. This family of catenaries is represented by the system of differential equations

$$
y^{\prime \prime}=\frac{1+y^{\prime 2}+z^{\prime 2}}{y}, \quad z^{\prime \prime}=0 .
$$

The generality of the corresponding variation problem is $\infty^{1 f(2)+1 y(1)}$.

The discussion of the non-separated case, IIa3, is, in the nature of the matter, not as simple or neat as that of the others. Nevertheless, every eventuality is followed out in the detailed considerations given in $\$ 13$. Even in the most favorable hypothetical possibility, the generality of the corresponding variation problem is certainly not higher than $\infty^{2 f(1)}$, as will be seen by a review of $\$ 13$.

Theorem IV. In Case IIb1' the given curve family is of extremal type and the generality of the corresponding variation problem is $\infty^{2 f(2)}$. The determination of $\phi$ depends on the solution of a complete system of three partial differential equations of first order for $\sigma\left(x, y, z, y^{\prime}, z^{\prime}\right)$ followed by the solution of a similar system for $\rho\left(x, y, z, y^{\prime}, z^{\prime}\right)$.

Example. $y^{\prime \prime}=z, z^{\prime \prime}=0$ (see $\left.\$ 15\right)$.

TheOREM V. In Case IIb1" no corresponding variation problem exists.

An example is given at the end of $\$ 15$.

Case IIb2 is discussed in $\$ 16$.

Theorem VI. In Case IIIa the solution of the inverse problem depends on a certain differential

$$
E_{1} d x+E_{2} d y+E_{3} d z+E_{4} d y^{\prime}+E_{5} d z^{\prime},
$$

whose coefficients $E_{i}$ are definite functions of the partial derivatives of $F, G$-indeed, rational functions having $D$ for denominator. In case this differential is exact, the corresponding variation problem exists and is essentially unique $\left({ }^{14}\right)$; in the contrary case, no corresponding variation problem exists.

Example. $y^{\prime \prime}=z^{2}, z^{\prime \prime}=y^{2}$. The corresponding integral is

$$
\int\left(y^{\prime} z^{\prime}+\frac{1}{3} y^{3}+\frac{1}{3} z^{3}\right) d x
$$

(14) Cf. formula (3.7) and the associated remarks. 
uniquely determined up to the possibility of the addition of an arbitrary exact differential and multiplication by an arbitrary constant factor (cf. formula 3.7).

Theorem VII. In Case IIIb no corresponding variation problem exists.

Example. $y^{\prime \prime}=y^{2}+z^{2}, z^{\prime \prime}=0$.

Theorem VIII. In Case IV no corresponding variation problem exists.

Example. $y^{\prime \prime}=y^{2}+z^{2}, z^{\prime \prime}=y$.

Part II. The fundamental differential system $\subseteq$

4. Derivation of $\mathfrak{S}$. The first step in our procedure is to make the solution of the original Euler-Lagrange equations (1.7) for the unknown function $\phi$ depend on an equivalent differential system $\subseteq$, more easily manageable, where the unknowns are the functions $\phi_{i j}$ defined by (1.9). We find it convenient to conduct the discussion for a general value of the dimensionality $n+1$, afterwards putting $n=2$.

Our derivation of the system $\mathfrak{S}$ is based on certain new identities which we establish involving the Euler expressions

These identities are

$$
\omega_{i} \equiv \frac{\partial \phi}{\partial y_{i}}-\frac{d}{d x} \frac{\partial \phi}{\partial y_{i}^{\prime}} \quad(i=1, \cdots, n) .
$$

where

$$
\begin{aligned}
\frac{\partial \omega_{i}}{\partial y_{j}^{\prime}}+\frac{\partial \omega_{j}}{\partial y_{i}^{\prime}} & +2 \frac{d}{d x} \phi_{i j}+\frac{\partial F_{k}}{\partial y_{j}^{\prime}} \phi_{i k}+\frac{\partial F_{k}}{\partial y_{i}^{\prime}} \phi_{j k}=0 \\
\frac{d}{d x}\left(\frac{\partial \omega_{i}}{\partial y_{j}^{\prime}}-\frac{\partial \omega_{j}}{\partial y_{i}^{\prime}}\right) & -2 \cdot\left(\frac{\partial \omega_{i}}{\partial y_{j}}-\frac{\partial \omega_{j}}{\partial y_{i}}\right)+\frac{1}{2} \frac{\partial F_{k}}{\partial y_{i}^{\prime}}\left(\frac{\partial \omega_{i}}{\partial y_{k}^{\prime}}+\frac{\partial \omega_{k}}{\partial y_{j}^{\prime}}\right) \\
& -\frac{1}{2} \frac{\partial F_{k}}{\partial y_{j}^{\prime}}\left(\frac{\partial \omega_{i}}{\partial y_{k}^{\prime}}+\frac{\partial \omega_{k}}{\partial y_{i}^{\prime}}\right)+A_{j k} \phi_{i k}-A_{i k} \phi_{j k}=0
\end{aligned}
$$

$$
A_{j k} \equiv \frac{d}{d x} \frac{\partial F_{k}}{\partial y_{j}^{\prime}}-2 \frac{\partial F_{k}}{\partial y_{j}}-\frac{1}{2} \frac{\partial F_{m}}{\partial y_{j}^{\prime}} \frac{\partial F_{k}}{\partial y_{m}^{\prime}} .
$$

Their verification may be left to the reader with the remark that here and frequently afterwards in this paper it is helpful to observe the following "alternating relations" between differential operators:

$$
\begin{aligned}
& \frac{\partial}{\partial y_{j}} \frac{d}{d x}-\frac{d}{d x} \frac{\partial}{\partial y_{j}}=\frac{\partial F_{k}}{\partial y_{j}} \frac{\partial}{\partial y_{k}^{\prime}}, \\
& \frac{\partial}{\partial y_{j}^{\prime}} \frac{d}{d x}-\frac{d}{d x} \frac{\partial}{\partial y_{j}^{\prime}}=\frac{\partial}{\partial y_{j}}+\frac{\partial F_{k}}{\partial y_{j}^{\prime}} \frac{\partial}{\partial y_{k}^{\prime}} .
\end{aligned}
$$


If the Euler-Lagrange equations $\omega_{i}=0$ are satisfied, then the preceding identities imply the following equations obeyed by the functions $\phi_{i j}$ :

$$
\begin{gathered}
\frac{d}{d x} \phi_{i j}+\frac{1}{2} \frac{\partial F_{k}}{\partial y_{j}^{\prime}} \phi_{i k}+\frac{1}{2} \frac{\partial F_{k}}{\partial y_{i}^{\prime}} \phi_{j k}=0, \\
A_{j k} \phi_{i k}-A_{i k} \phi_{j k}=0 .
\end{gathered}
$$

We also have evidently from (1.9):

$$
\frac{\partial \phi_{i j}}{\partial y_{k}^{\prime}}=\frac{\partial \phi_{i k}}{\partial y_{j}^{\prime}}
$$

and we take explicit notice of the symmetry of $\phi_{i j}$ in its indices:

$$
\phi_{i j}=\phi_{i i}
$$

The differential system in $\phi_{i j}$ consisting of the equations (4.7)-(4.10) together with the inequation (1.8):

$$
\text { Det }\left|\phi_{i j}\right| \neq 0
$$

is our fundamental differential system $\subseteq$.

It is to be observed that all the equations of $\subseteq$ are linear.

5. Equivalence of the system $\subseteq$ to the Euler-Lagrange equations. Conversely, let $\phi_{i j}$ be any system of functions of $x, y_{k}, y_{k}^{\prime}$ which obeys the system S.

Then (4.9) implies the existence of functions

$$
\phi_{i}=\int \phi_{i j} d y_{j}^{\prime},
$$

since the differential under the integral sign is exact. We have $\partial \phi_{i} / \partial y_{j}^{\prime}=\phi_{i j}$; therefore by (4.10), $\partial \phi_{i} / \partial y_{j}^{\prime}=\partial \phi_{j} / \partial y_{i}^{\prime}$, from which it is possible to conclude the exactness of the differential $\phi_{i} d y_{i}^{\prime}$ and the existence of a function

$$
\phi=\int \phi_{i} d y_{i}^{\prime}
$$

Evidently the functions $\phi_{i j}$ are the second partial derivatives of $\phi$, as required by (1.9).

The existence of a function $\phi$ whose second partial derivatives $\partial^{2} \phi / \partial y_{i}^{\prime} \partial y_{j}^{\prime}$ are precisely the functions $\phi_{i j}$ being thus assured, it is next evident that $\phi$ is determined only up to the addition of an arbitrary linear function of the $y_{k}{ }^{\prime}$ :

$$
\phi=\bar{\phi}+\lambda_{0}+\lambda_{k} y_{k}^{\prime}
$$

where $\bar{\phi}$ denotes any fixed determination and $\lambda_{0}, \lambda_{k}$ are functions of $x, y_{j}$. 
Let functions $\bar{\omega}_{i}$ be deduced from any fixed determination $\bar{\phi}$ by formula (4.1). Then by the identities $(4.2,3)$ and the equations $(4.7,8)$, it follows that

$$
\begin{gathered}
\frac{\partial \bar{\omega}_{i}}{\partial y_{j}^{\prime}}+\frac{\partial \bar{\omega}_{j}}{\partial y_{i}^{\prime}}=0 \\
\frac{d}{d x}\left(\frac{\partial \bar{\omega}_{i}}{\partial y_{j}^{\prime}}-\frac{\partial \bar{\omega}_{j}}{\partial y_{i}^{\prime}}\right)-2\left(\frac{\partial \bar{\omega}_{i}}{\partial y_{j}}-\frac{\partial \bar{\omega}_{j}}{\partial y_{i}}\right)=0 .
\end{gathered}
$$

Applying $\partial / \partial y_{k}^{\prime}$ to (5.4), we get

$$
\frac{\partial^{2} \bar{\omega}_{i}}{\partial y_{j}^{\prime} \partial y_{k}^{\prime}}+\frac{\partial^{2} \bar{\omega}_{j}}{\partial y_{i}^{\prime} \partial y_{k}^{\prime}}=0
$$

and cyclic permutation of $i, j, k$ gives three equations of this type, wherefrom

$$
\frac{\partial^{2} \bar{\omega}_{i}}{\partial y_{j}^{\prime} \partial y_{k}^{\prime}}=0 \text {. }
$$

This expresses that $\bar{\omega}_{i}$ is linear in the variables $y_{k}^{\prime}$ :

$$
\bar{\omega}_{i}=\alpha_{i k} y_{k}^{\prime}+\beta_{i},
$$

where $\alpha_{i k}, \beta_{i}$ are functions of $x, y_{j}$. Formula (5.4) then implies the skew-symmetry of $\alpha_{i j}$ :

$$
\alpha_{i j}+\alpha_{j i}=0 .
$$

Substituting (5.6) in (5.5), we find

$$
\begin{aligned}
& \frac{\partial \alpha_{i j}}{\partial y_{k}}+\frac{\partial \alpha_{k i}}{\partial y_{j}}+\frac{\partial \alpha_{j k}}{\partial y_{i}}=0, \\
& \frac{\partial \alpha_{i j}}{\partial x}+\frac{\partial \beta_{i}}{\partial y_{i}}-\frac{\partial \beta_{i}}{\partial y_{j}}=0 .
\end{aligned}
$$

If we extend the range $1, \cdots, n$ of the indices $i, j$ in $\alpha_{i j}$ to include the index 0 by defining $\alpha_{i 0}=\beta_{i}, \alpha_{0 i}=-\beta_{i}, \alpha_{00}=0$ (which agrees with (5.7)), then (5.9) becomes

$$
\frac{\partial \alpha_{i j}}{\partial x}+\frac{\partial \alpha_{j 0}}{\partial y_{i}}+\frac{\partial \alpha_{0 i}}{\partial y_{j}}=0 ;
$$

we may say therefore that (5.8) holds for the range $0,1, \cdots, n$ of the indices (provided, further, that $y_{0}$ is identified with $x$ ).

Let now $\bar{\phi}$ be replaced by the general determination $\phi$ according to (5.3). Then, as is readily calculated from (4.1), $\bar{\omega}_{i}$ is transformed into

$$
\omega_{i}=\bar{\omega}_{i}-\left(\frac{\partial \lambda_{i}}{\partial x}-\frac{\partial \lambda_{0}}{\partial y_{i}}\right)-\left(\frac{\partial \lambda_{i}}{\partial y_{k}}-\frac{\partial \lambda_{k}}{\partial y_{i}}\right) y_{k}{ }^{\prime} .
$$


By the substitution (5.6), we see that $\omega_{i}=0$ if and only if

$$
\begin{array}{lrl}
\frac{\partial \lambda_{i}}{\partial y_{k}}-\frac{\partial \lambda_{k}}{\partial y_{i}}=\alpha_{i k}, & (i, k=1, \cdots, n), \\
\frac{\partial \lambda_{i}}{\partial x}-\frac{\partial \lambda_{0}}{\partial y_{i}}=\beta_{i}=\alpha_{i 0}, & (i=1, \cdots, n) .
\end{array}
$$

These conditions express that the curl of the unknown vector $\lambda=\left(\lambda_{0}, \lambda_{1}, \cdots, \lambda_{n}\right)$ shall be the known skew-symmetric tensor $\alpha_{\rho \sigma}$ $(\rho, \sigma=0,1, \cdots, n)$. It is well-known that the established relations (5.8), (5.10) are exactly necessary and sufficient in order that such a vector $\lambda$ exist. $\lambda$ is then determined up to the addition of an arbitrary gradient $\left(\partial \nu / \partial x, \partial \nu / \partial y_{1}, \cdots, \partial \nu / \partial y_{n}\right)$ where $\nu$ is any function of $x, y_{j}$. That is, according to (5.3), $\phi$ is determined $u p$ to the addition of an arbitrary total derivative as to $x$ :

$$
\frac{d}{d x} \nu\left(x, y_{k}\right)=\frac{\partial \nu}{\partial x}+\frac{\partial \nu}{\partial y_{k}} y_{k}^{\prime}
$$

As we have already remarked, it is evident $a$ priori that we could not expect to determine $\phi$ any more closely than this, since the addition of an exact differential $d \nu\left(x, y_{k}\right)$ to $\phi d x$ has no effect on the extremals of $\int \phi d x$.

In summary, we may state the following

Proposition $\left({ }^{15}\right)$. To every solution $\phi_{i j}$ of the system $\subseteq$ there corresponds a solution $\phi$ of the Euler-Lagrange equations having $\phi_{i j}$ as the system of its second partial derivatives as to the $y^{\prime \prime} s: \partial^{2} \phi / \partial y_{i}^{\prime} \partial y_{j}^{\prime}=\phi_{i j}$. For a given solution $\phi_{i j}$, the function $\phi$ can be found by successive quadratures $\left({ }^{16}\right)$, and is uniquely determined except for the inevitable addition of an arbitrary total derivative $d \nu\left(x, y_{k}\right) / d x$.

In other words, the inverse problem of the calculus of variations is precisely equivalent to the solution for $\phi_{i j}$ of the differential system $\subseteq$.

\section{Part III ( $\$ 6$ ). OUtLine of the general theory of DIFFERENTIAL SYSTEMS}

The essence of this paper consists in an application of the general theory of differential systems, as presented in the standard treatises $\left({ }^{17}\right)$, to the particular system $\subseteq$. Referring the reader to these works, we shall presuppose the vocabulary, ideas, and facts of this theory. However, for purposes of ex-

(15) This proposition, as well as the proof here given (essentially), is contained in the papers of Davis referred to in $\$ 2$.

(16) The determination of a vector, given its curl, can be effected by a series of quadratures.

(17) These are, principally: C. Riquier, Les Systèmes d'Équations aux Dérivées Partielles, Paris, 1910; M. Janet, Leçons sur les Systèmes d'Équations aux Dérivées Partielles, Paris, 1929; J. F. Ritt, Differential Equations from the Algebraic Standpoint, American Mathematical Society 
position-particularly, to render the sequel intelligible to those not well acquainted with the theory of differential systems, as developed principally by Riquier-it seems desirable to give here a brief outline of the procedure for testing the consistency of any given differential system and, in case a solution exists, for obtaining an equivalent completely integrable system which will give this solution. The application of the general theory to our particular case is very considerably simplified by the circumstance that $\subseteq$ is linear.

By a differential system we understand any finite number of equations in which appear any finite number $n$ of independent variables $x_{j}$, and any finite number $m$ of unknown functions $u_{i}$ of these variables, together with a certain finite number of partial derivatives of the functions $u_{i}$ with respect to the $x_{j}$ up to any order. Each member of each equation is supposed to be an analytic function of the variables $x_{j}, u_{i}$ and those partial derivatives of the $u_{i}$ which it involves effectively, this function being regular, that is, representable as a power-series, about certain initial values of all these variables. The system may also contain a certain finite number of inequations; that is, certain analytic functions of $x_{j}, u_{i}$ and some of the partial derivatives of the $u_{i}$ may be required to be $\neq 0$.

If a differential system is in a form solved for certain derivatives of the unknown functions $u_{i}$, we term any derivative of a function $u_{i}$ which can be obtained by differentiation of a first member to any order with respect to the independent variables $x_{j}$ a principal derivative. (This definition is interpreted to include the first members themselves among the principal derivatives, as their own derivatives of zeroth order.) All other partial derivatives of the functions $u_{i}$ are termed parametric.

Riquier introduced the device of cotes. With each independent variable $x_{j}$ is associated a composite integer with any fixed number $s$ of components: $c_{j} \equiv\left(c_{j 1}, c_{j 2}, \cdots, c_{j s}\right)$, where each $c_{j k}$ is an in teger $\geqq 0$; this composite integer is what is called the "cote" of $x_{j}$. Similarly a cote is assigned to each unknown function $u_{i}: c_{i}^{\prime} \equiv\left(c_{i 1}^{\prime}, c_{i 2}{ }^{\prime}, \cdots, c_{i s}{ }^{\prime}\right)$. The cote of any partial derivative

$$
\frac{\partial^{r_{1}+r_{2}+\cdots+r_{n} u_{i}}}{\partial x_{1}^{r_{1}} \partial x_{2}^{r_{2}} \cdots \partial x_{n}^{r_{n}}}
$$

is then, by definition,

$$
\Gamma \equiv\left(\gamma_{1}, \gamma_{2}, \cdots, \gamma_{s}\right)
$$

where $\gamma_{k}=c_{i k}^{\prime}+r_{1} c_{1 k}+r_{2} c_{2 k}+\cdots+r_{n} c_{n k}$.

Colloquium Publications, vol. 14, New York, 1932; J. M. Thomas, Differential Systems, American Mathematical Society Publications, vol. 21, New York, 1937.

See also an exposition of the Riquier theory by Thomas, Annals of Mathematics, (2), vol. 30 (1929), p. 285 et seq.

Particularly relevant from our viewpoint are: Riquier, chap. 7; Janet, pp. 74-75; Ritt, chap. 9; Thomas, $\S 36,37,75,76$. 
All the derivatives of all the unknown functions $\left({ }^{18}\right)$ are arranged according to their cotes in "dictionary order," that is, given any two derivatives $\delta u_{i}$, $\delta^{\prime} u_{j}$ with respective cotes

$$
\Gamma \equiv\left(\gamma_{1}, \gamma_{2}, \cdots, \gamma_{s}\right), \quad \Gamma^{\prime} \equiv\left(\gamma_{1}^{\prime}, \gamma_{2}^{\prime}, \cdots, \gamma_{s}^{\prime}\right),
$$

$\delta u_{i}$ is called "higher" than $\delta^{\prime} u_{j}$ if the first of the differences

$$
\gamma_{1}-\gamma_{1}^{\prime}, \gamma_{2}-\gamma_{2}^{\prime}, \cdots, \gamma_{8}-\gamma_{s}^{\prime}
$$

which is not zero is positive; if the first of these nonzero differences is negative, $\delta u_{i}$ is called "lower" than $\delta^{\prime} u_{j}$, or $\delta^{\prime} u_{j}$ higher than $\delta u_{i}$. In case $\Gamma=\Gamma^{\prime}$, that is, $\gamma_{k}=\gamma_{k}^{\prime}$ for $k=1, \cdots, s$, no distinction of higher and lower is created between $\delta u_{i}$ and $\delta^{\prime} u_{j}$. The cotes can always be assigned so that this eventuality never occurs, and the ordering of derivatives produced by the cotes is then said to be complete.

It is an essential condition, always supposed verified in the Riquier theory, that the first cote $c_{j 1}$ of each independent variable $x_{j}$ be equal to 1 .

Riquier terms a differential system $\subseteq$ orthonomic if it obeys the following three conditions:

$1^{\circ}$ it is solved for distinct derivatives of the unknown functions;

$2^{\circ}$ no second member contains any principal derivative effectively;

$3^{\circ}$ cotes have been assigned, in accordance with the stipulation $c_{j 1}=1$, so that each first member is higher than any derivative which appears effectively in the corresponding second member.

The prolonged system $\widetilde{\varsigma}^{\prime}$ of any given differential system $\subseteq$ is the infinite system which is derived from $\mathfrak{S}$ by subjecting each of its equations to every possible differentiation with respect to the independent variables $x_{j}$ : $\partial^{r_{1}+r_{2}+\cdots+r_{n}} / \partial x_{1}^{r_{1}} \partial x_{2}^{r_{2}} \cdots \mid \partial x_{n}^{r_{n}}$. In this differentiation each member of each equation of $\subseteq$ is considered as a composite function of the $x_{j}$ through the intermediary of those partial derivatives of the functions $u_{i}$ which appear in this member, and the differentiation is effected according to the composite function rule.

The important idea of a passive system $\subseteq$ is defined as follows. $\subseteq$ is called "passive" if its prolonged system $\mathfrak{S}^{\prime}$ is equivalent to a system $\mathfrak{S}^{\prime \prime}$ which consists of a unique expression for each principal derivative in terms of a finite number of parametric derivatives. This means that the equations of $\mathfrak{S}^{\prime}$ will be satisfied identically in the parametric derivatives if we substitute for each principal derivative its expression from $\mathfrak{\complement}^{\prime \prime}$.

A fundamental theorem of the Riquier theory is that every differential system which is passive and orthonomic is completely integrable. The meaning of this is that a unique solution of the given differential system exists which

(18) The unknown functions will be regarded throughout as their own derivatives of zeroth order. 
corresponds to initial data of the following nature: the values of all the parametric derivatives are assigned arbitrarily for any fixed initial values $x_{j}^{(0)}$ of the independent variables $x_{j}$, provided only that we have convergence of that part of the Taylor series of each unknown function $u_{i}$ about $x_{j}^{(0)}$ which involves these parametric derivatives as coefficients. In other words, the initial values of all principal derivatives may be regarded as having been put equal to zero to give this "initial determination" of the functions $u_{i}$.

The same initial determination may be represented in the manner now to be described. It is a fact, proved in the general theory, that all parametric derivatives may be obtained from a certain finite number among them, called a complete set, as follows. With respect to each parametric derivative of the complete set, we distinguish the independent variables $x_{j}$ according to a certain rule as multipliers and non-multipliers. Then each parametric derivative is obtainable from a unique one of the complete set by a unique differentiation with respect to its multipliers. From this, one can infer that the initial assignment of all parametric derivatives subject to the stated convergence condition is equivalent to assigning to each partial derivative $\delta u_{i}$ of the complete set an arbitrary convergent Taylor series in the multipliers $x_{j}$ of $\delta u_{i}$ (power-series $P\left(x_{j}-x_{j}^{(0)}\right)$ in the quantities $\left.x_{j}-x_{j}^{(0)}\right)$ and requiring $\delta u_{i}$ to reduce to this series when the non-multipliers $x_{k}$ are set equal to their initial values: $\delta u_{i}=P\left(x_{j}-x_{j}^{(0)}\right)$ for each $x_{k}=x_{\mathbf{k}}^{(0)}$. This type of initial condition then fixes a solution of the given differential system uniquely.

One may consult J. M. Thomas, loc. cit., $\$ \$ 75,76$, or Ritt, loc. cit., p. 139 for the notion of complete set, both of principal and of parametric derivatives, and for the definition of multipliers and non-multipliers of each derivative belonging to a complete set. It is convenient here, as throughout the theory of differential systems, to employ an obvious isomorphism between partial derivatives of a given function $u\left(x_{1}, x_{2}, \cdots, x_{n}\right)$ and algebraic monomials in the variables $x_{j}$ : thus $\partial^{r_{1}+r_{2}}+\cdots+r_{n} u / \partial x_{1}^{\tau_{1}} \partial x_{2}^{r_{2}} \cdots \partial x_{n}^{\tau_{n}}$ corresponds to the monomial $x_{1}^{\tau_{1}} x_{2}^{\tau_{2}} \cdots x_{n}^{\tau_{n}}$, and any successive operations of differentiation with respect to the independent variables $x_{j}$ correspond to multiplication of the associated monomials.

The importance of the passive nature of a differential system makes it essential to have a criterion for the passivity of a given orthonomic differential system $\subseteq$. Each equation of the prolonged system $\Im^{\prime}$ expresses a certain principal derivative in terms of parametric derivatives and of principal derivatives of lower cote; this follows from the orthonomic nature of $\mathfrak{S}$, which can be proved to extend to the prolonged system $\Im^{\prime}$. The expressions for the principal derivatives thus furnished by $\varsigma^{\prime}$ are said to be direct. It is much to be emphasized that a given principal derivative may occur as first member in many different equations of $\varsigma^{\prime}$, that is, be obtainable in many different ways by differentiation of first members of $\subseteq$. For example, if $\partial^{3} u / \partial x \partial y^{2}$, $\partial^{3} u / \partial x^{2} \partial y, \partial u / \partial x$ are first members of $\subseteq$, the derivative $\partial^{5} u / \partial x^{3} \partial y^{2}$ may 
be obtained from these respectively by the differentiations $\partial^{2} / \partial x^{2}, \partial^{2} / \partial x \partial y$, $\partial^{4} / \partial x^{2} \partial y^{2}$. Accordingly, any principal derivative may, and generally will, have various direct expressions.

Imagine the principal derivatives arranged in order of cote from lower to higher as first, second, third, $\cdots: P_{1}, P_{2}, P_{3}, \cdots$. Then the lowest principal derivative $P_{1}$ must, as can easily be shown, be a first member of $\mathfrak{S}$, and has therefore, by condition $1^{\circ}$ for an orthonomic system, a unique expression in terms of parametric derivatives exclusively (the independent variables $x_{j}$ are supposed to figure throughout as parameters). Consider next the second lowest principal derivative $P_{2}$; each of its direct expressions involves, besides parametric derivatives, only the lowest principal derivative $P_{1}$; if the expression just mentioned for this is substituted, then a certain number of expressions are obtained for $P_{2}$ in terms of parametric derivatives exclusively-these are termed the ultimate expressions of $P_{2}$. Next, the third lowest principal derivative $P_{3}$ has a certain number of direct expressions in terms of parametric derivatives and the two lower principal derivatives $P_{1}, P_{2}$. If in each of these direct expressions for $P_{3}$ we substitute the unique ultimate expression for $P_{1}$ and, in succession, all the different ultimate expressions already obtained for $P_{2}$, we get a certain number of ultimate expressions for $P_{3}$ in terms of parametric derivatives exclusively. Continuing step-by-step in this way, we obtain for each principal derivative $P_{k}$ a certain number of ultimate expressions in terms of parametric derivatives.

A criterion for the passivity of the given differential system is that all the various ultimate expressions of each principal derivative $P_{k}$ shall be identical.

This criterion may be considerably simplified by basing the test for passivity on only a certain finite number of principal derivatives, called cardinal derivatives, instead of the infinite set of all of them. If $\partial^{r_{1}+r_{2}+\cdots+r_{n}} u_{i} / \partial x_{1}^{r_{1}} \partial x_{2}^{r_{2}} \cdots \partial x_{n}^{r_{n}}$ and $\partial^{s_{1}+s_{2}+\cdots+s_{n}} u_{i} / \partial x_{1}^{s_{1}} \partial x_{2}^{s_{2}} \cdots \partial x_{n}^{s_{n}}$ are any two first members of $\subseteq$ which are partial derivatives of the same unknown function $u_{i}$, then the corresponding cardinal derivative is, by definition, $\partial^{t_{1}+t_{2}+\cdots+t_{n}} u_{i} / \partial x_{1}^{t_{1}} \partial x_{2}^{t_{2}} \cdots \partial x_{n}^{t_{n}}$ where each $t_{j}$ is the greater of $r_{j}$ and $s_{j}\left({ }^{19}\right)$. Then, as proved by Riquier, the following is a necessary and sufficient condition for the passivity of $\mathfrak{S}$ : the various ultimate expressions for each cardinal derivative shall be identical.

This is the form of test which will be used in the applications that follow.

If $\subseteq$ is not passive, the application of the preceding criterion, that is, the equating of every two different ultimate expressions for a cardinal derivative, will lead to relations among the parametric derivatives which are not verified identically. By solving these for the parametric derivatives of highest cote $\left({ }^{20}\right)$, we obtain an orthonomic differential system $\Im_{1}$ equivalent to $\subseteq$ in the sense that any solution of the one must be a solution of the other.

(19) In terms of the isomorphism between partial derivatives and monomials mentioned earlier in this section, cardinal derivative corresponds to least common multiple.

$\left.{ }^{(20}\right)$ Cf. Janet, loc. cit., p. 75. 
The passage from $\mathfrak{\subseteq}$ to $\mathfrak{S}_{1}$ clearly enlarges the class of principal derivatives and narrows that of parametric derivatives.

If we next apply our passivity test to $\Im_{1}$, then either it is verified that $\Im_{1}$ is passive, or else new equations not identically satisfied are obtained among parametric derivatives of $\mathfrak{S}_{1}$. In the latter case a system $\mathfrak{S}_{2}$ is derived as before, equivalent to $\subseteq$ and $\mathfrak{S}_{1}$, and with a still wider class of principal derivatives and a narrower one of parametric derivatives.

It is a standard fact of the theory of differential systems that this process, accompanied by successive enlargement of the class of principal derivatives and restriction of the class of parametric derivatives, can go on for only a finite number $k$ of steps. That this is so is a direct consequence of the following theorem due to Riquier: if in a sequence of monomials $x_{1}^{\gamma_{1}} x_{2}^{\tau_{2}} \cdots x_{n}^{\tau_{n}}$ (each $r_{j}$ an integer $\geqq 0)$ no monomial is a multiple of a predecessor, the sequence can contain only a finite number of terms. (Cf. Janet, loc. cit., p. 11; Ritt, loc. cit., p. 135; and refer also to the isomorphism between partial derivatives and monomials mentioned earlier in this section.)

At the end of the finite process just described, either the conditions for passivity are found to be verified, or else we obtain a non-identical relation involving only the independent variables, or a relation contradicting one of the specified inequations of $\mathfrak{S}$. In the latter two eventualities the system $\mathfrak{S}$ is inconsistent with itself, or has no solutions; in the former case we obtain a passive orthonomic system [ $\subseteq]=\Im_{k}$ equivalent to $\subseteq$, which has therefore a unique solution corresponding to initial data of the type previously described; that is, [ऽ] is completely integrable.

The main body of this paper, which follows, is concerned with applying the general theory just outlined to the particular differential system $\mathfrak{S}$ on which depends the inverse problem of the calculus of variations.

\section{Part IV. Solution of the differential system $\mathfrak{S}$}

7. The system $\subseteq$ in the three-dimensional case. We have derived the differential system $\subseteq$ in $\S \S 4,5$ for a general value of the dimensionality $n+1$. For the three-dimensional inverse problem, which alone will be considered in this paper, $n=2$, and we adopt the notation $\left(x, y_{1}, y_{2}, y_{1}^{\prime}, y_{2}^{\prime}\right)=\left(x, y, z, y^{\prime}, z^{\prime}\right)$, $\left(F_{1}, F_{2}\right)=(F, G)$, so that, as already written in $\$ 1$, the differential equations of the given curve family are

$$
y^{\prime \prime}=F\left(x, y, z, y^{\prime}, z^{\prime}\right), \quad z^{\prime \prime}=G\left(x, y, z, y^{\prime}, z^{\prime}\right),
$$

while the variation problem sought for is

$$
\int \phi\left(x, y, z, y^{\prime}, z^{\prime}\right) d x=\min .
$$

For the functions $\phi_{i j}(i, j=1,2)$ we write 


$$
L=\phi_{y^{\prime} y^{\prime}}, \quad M=\phi_{y^{\prime} z^{\prime}}, \quad N=\phi_{z^{\prime} z^{\prime}}
$$

The differential operator (1.6) is now

$$
\frac{d}{d x} \equiv \frac{\partial}{\partial x}+y^{\prime} \frac{\partial}{\partial y}+z^{\prime} \frac{\partial}{\partial z}+F \frac{\partial}{\partial y^{\prime}}+G \frac{\partial}{\partial z^{\prime}} .
$$

By comparison with (4.7)-(4.11), we see that the fundamental differential system $\subseteq$ now consists of the following six linear equations and quadratic inequation in $L, M, N$ as unknown functions:

$$
\begin{aligned}
\frac{d L}{d x}+F_{y^{\prime}} L+G_{y^{\prime}} M & =0, \\
\frac{d M}{d x}+\frac{1}{2} F_{z^{\prime}} L+\frac{1}{2}\left(F_{y^{\prime}}+G_{z^{\prime}}\right) M+\frac{1}{2} G_{y^{\prime}} N & =0, \\
\frac{d N}{d x}+F_{z^{\prime}} M+G_{z^{\prime}} N & =0, \\
A L+B M+C N & =0, \\
L_{z^{\prime}} & =M_{y^{\prime}}, \\
N_{y^{\prime}} & =M_{z^{\prime}}, \\
L N-M^{2} & \neq 0 .
\end{aligned}
$$

In the purely algebraic equation $\left(7.5_{4}\right)$ of the system, the coefficients $A, B, C$ are, by (4.4),

$$
\begin{aligned}
& A=\frac{d}{d x} F_{z^{\prime}}-2 F_{z}-\frac{1}{2} F_{z^{\prime}}\left(F_{y^{\prime}}+G_{z^{\prime}}\right), \\
& B=-\frac{d}{d x} F_{y^{\prime}}+\frac{d}{d x} G_{z^{\prime}}+2\left(F_{y}-G_{z}\right)+\frac{1}{2}\left(F_{y^{\prime}}^{2}-G_{z^{\prime}}^{2}\right), \\
& C=-\frac{d}{d x} G_{y^{\prime}}+2 G_{y}+\frac{1}{2} G_{y^{\prime}}\left(F_{y^{\prime}}+G_{z^{\prime}}\right) .
\end{aligned}
$$

Applying successively to (7.54) the operator $d / d x$, and using the first three equations of (7.5), we obtain the following two additional purely algebraic linear equations in $L, M, N$ :

$$
\begin{aligned}
& A_{1} L+B_{1} M+C_{1} N=0, \\
& A_{2} L+B_{2} M+C_{2} N=0 .
\end{aligned}
$$

Here $A_{1}, B_{1}, C_{1}$ are derived from $A, B, C$ by the formulas 


$$
\begin{aligned}
& A_{1}=\frac{d A}{d x}-F_{y^{\prime}} A-\frac{1}{2} F_{z^{\prime}} B, \\
& B_{1}=\frac{d B}{d x}-G_{y^{\prime}} A-\frac{1}{2}\left(F_{y^{\prime}}+G_{z^{\prime}}\right) B-F_{z^{\prime}} C, \\
& C_{1}=\frac{d C}{d x}-\frac{1}{2} G_{y^{\prime}} B-G_{z^{\prime}} C,
\end{aligned}
$$

while $A_{2}, B_{2}, C_{2}$ are derived from $A_{1}, B_{1}, C_{1}$ by the same formulas (recursion).

Since $(L, M, N) \neq(0,0,0)$-for the contrary would be inconsistent with the last condition of (7.5) - we infer from the system of linear equations $\left(7.5_{4}\right)$, (7.7), (7.8) the following property of all extremal curve families:

$$
\left|\begin{array}{lll}
A & B & C \\
A_{1} & B_{1} & C_{1} \\
A_{2} & B_{2} & C_{2}
\end{array}\right|=0 .
$$

This necessary condition gives a very easy way of constructing nonextremal curve families, namely, by the nonvanishing of the determinant just written. The readily verified example given in (1.11) was found in this manner $\left({ }^{21}\right)$.

8. Case I. When the fundamental matrix is of rank zero. As stated in $\S 3$, the principal basis of our classification into cases is the rank of the matrix

$$
\Delta \equiv\left\|\begin{array}{lll}
A & B & C \\
A_{1} & B_{1} & C_{1} \\
A_{2} & B_{2} & C_{2}
\end{array}\right\| .
$$

The first case to consider is that of rank zero, that is, when

$$
A=0, \quad B=0, \quad C=0 .
$$

It is evident by the recursion formulas (7.9) that every element of $\Delta$ is then zero; that is, the rank of $\Delta$ is indeed zero.

The fourth equation (7.5) now disappears identically, so that the system $\subseteq$ is now constituted by the remaining five equations and the inequation of (7.5). We proceed to consider $\mathfrak{S}$ from the standpoint of the Riquier theory.

This is the place to make the following preliminary remark. In the theory of the system $\subseteq$ we may use $d / d x$ instead of $\partial / \partial x$, that is, we may take as fundamental the differential operators $d / d x, \partial / \partial y, \partial / \partial z, \partial / \partial y^{\prime}, \partial / \partial z^{\prime}$ instead of $\partial / \partial x, \partial / \partial y, \partial / \partial z, \partial / \partial y^{\prime}, \partial / \partial z^{\prime}$. In doing this, it is important to have always in mind the following "alternating relations":

(21) Cf. §18. 


$$
\begin{aligned}
& \frac{\partial}{\partial y} \frac{d}{d x}-\frac{d}{d x} \frac{\partial}{\partial y}=F_{y} \frac{\partial}{\partial y^{\prime}}+G_{y} \frac{\partial}{\partial z^{\prime}} \\
& \frac{\partial}{\partial z} \frac{d}{d x}-\frac{d}{d x} \frac{\partial}{\partial z}=F_{z} \frac{\partial}{\partial y^{\prime}}+G_{z} \frac{\partial}{\partial z^{\prime}} \\
& \frac{\partial}{\partial y^{\prime}} \frac{d}{d x}-\frac{d}{d x} \frac{\partial}{\partial y^{\prime}}=\frac{\partial}{\partial y}+F_{y^{\prime}} \frac{\partial}{\partial y^{\prime}}+G_{y^{\prime}} \frac{\partial}{\partial z^{\prime}} \\
& \frac{\partial}{\partial z^{\prime}} \frac{d}{d x}-\frac{d}{d x} \frac{\partial}{\partial z^{\prime}}=\frac{\partial}{\partial z}+F_{z^{\prime}} \frac{\partial}{\partial y^{\prime}}+G_{z^{\prime}} \frac{\partial}{\partial z^{\prime}}
\end{aligned}
$$

Then, for instance, instead of employing such a passivity condition as

$$
\frac{\partial}{\partial z^{\prime}} L_{x}=\frac{\partial}{\partial x} L_{z^{\prime}}
$$

we use

$$
\frac{\partial}{\partial z^{\prime}} \frac{d L}{d x}-\frac{d}{d x} L_{z^{\prime}}=L_{z}+F_{z^{\prime}} L_{y^{\prime}}+G_{z^{\prime}} L_{z^{\prime}}
$$

and likewise in all similar cases.

We now adopt the following system of cotes for the independent variables and unknown functions of $\mathfrak{S}$ :

\begin{tabular}{l|l|l|l|l|l|l|l}
\hline \hline$x$ & $y$ & $z$ & $y^{\prime}$ & $z^{\prime}$ & $L$ & $M$ & $N$ \\
\hline 1 & 1 & 1 & 1 & 1 & 0 & 0 & 0 \\
0 & 0 & 0 & 0 & 0 & 3 & 1 & 2 \\
1 & 0 & 0 & 0 & 0 & 0 & 0 & 0 \\
0 & 1 & 0 & 0 & 0 & 0 & 0 & 0 \\
0 & 0 & 1 & 0 & 0 & 0 & 0 & 0 \\
0 & 0 & 0 & 1 & 0 & 0 & 0 & 0 \\
0 & 0 & 0 & 0 & 1 & 0 & 0 & 0 \\
\hline
\end{tabular}

This is equivalent to arranging all the derivatives of $L, M, N$ first with regard to their total order in all the independent variables, then with regard to the unknown function in the order $L, N, M$ (note that $M$ is last), then as to the order in $x$, in $y$, in $z$, in $y^{\prime}$, in $z^{\prime}$. The cote of $x$ is thought of as associated with the operator $d / d x$ rather than $\partial / \partial x$.

With these cotes, the system $\mathfrak{S}$ is seen to be orthonomic, for it has the three characteristic properties stated in $\$ 6$.

There are two passivity tests to be applied, the first associated with (8.5), the second with 


$$
\frac{\partial}{\partial y^{\prime}} \frac{d N}{d x}-\frac{d}{d x} N_{y^{\prime}}=N_{y}+F_{y^{\prime}} N_{y^{\prime}}+G_{y^{\prime}} N_{z^{\prime}}
$$

In all such passivity tests involving the operation $d / d x$ we may proceed systematically as follows. The first three equations of (7.5) represent $d / d x$ of $L, M, N$ as linear expressions in $L, M, N$. By use of the alternating relations (8.3), we may then represent $d / d x$ of any first partial derivative of $L, M, N$ as a linear expression in these partial derivatives and $L, M, N$ themselves, that is, as a linear differential expression of the first order in $L, M, N$. Continuing step-by-step in this way, we can represent $d / d x$ of any $k$ th order partial derivative of $L, M, N$ as a linear differential expression of $k$ th order in $L, M, N$.

Accordingly, by applying $d / d x$ to any linear differential relation in $L, M$, $N$ we obtain another one of the same order. By eliminating from this all principal derivatives, as we may do, we have a relation among parametric derivatives which is a consequence of the original differential relation. According to the general theory of $\S 6$, this new relation must be satisfied identically for a passivity condition; otherwise, it must be adjoined to the given differential system so as to form an extended system.

Let us then apply $d / d x$ to the fifth equation (7.5), obtaining

$$
\frac{d}{d x} L_{z^{\prime}}=\frac{d}{d x} M_{y^{\prime}}
$$

By (8.5) and the first equation of (7.5),

$$
\frac{d}{d x} L_{z^{\prime}}=\frac{\partial}{\partial z^{\prime}}\left(-F_{y^{\prime}} L-G_{y^{\prime}} M\right)-L_{z}-F_{z^{\prime}} L_{y^{\prime}}-G_{z^{\prime}} L_{z^{\prime}}
$$

Similarly,

$$
\frac{d}{d x} M_{y^{\prime}}=\frac{\partial}{\partial y^{\prime}}\left[-\frac{1}{2} F_{z^{\prime}} L-\frac{1}{2}\left(F_{y^{\prime}}+G_{z^{\prime}}\right) M-\frac{1}{2} G_{y^{\prime}} N\right]-M_{y}-F_{y^{\prime}} M_{y^{\prime}}-G_{y^{\prime}} M_{z^{\prime}}
$$

Equating the last two expressions, we find, after writing $L_{z^{\prime}}=M_{y^{\prime}}, N_{y^{\prime}}=M_{z^{\prime}}$, the following relation among parametric derivatives of $\mathfrak{S}$ :

$$
\begin{aligned}
L_{z}=M_{y} & -\frac{1}{2} F_{z^{\prime}} L_{y^{\prime}}+\frac{1}{2}\left(F_{y^{\prime}}-G_{z^{\prime}}\right) M_{y^{\prime}}+\frac{1}{2} G_{y^{\prime}} M_{z^{\prime}} \\
& -\frac{1}{2} F_{y^{\prime} z^{\prime}} L+\frac{1}{2}\left(F_{y^{\prime} y^{\prime}}-G_{y^{\prime} z^{\prime}}\right) M+\frac{1}{2} G_{y^{\prime} y^{\prime}} N .
\end{aligned}
$$

Treating the sixth equation of (7.5) in a similar way:

$$
\frac{d}{d x} N_{y^{\prime}}=\frac{d}{d x} M_{z^{\prime}}
$$

we get 


$$
\begin{aligned}
N_{y}=M_{z} & +\frac{1}{2} F_{z^{\prime}} M_{y^{\prime}}-\frac{1}{2}\left(F_{y^{\prime}}-G_{z^{\prime}}\right) M_{z^{\prime}}-\frac{1}{2} G_{y^{\prime}} N_{z^{\prime}} \\
& +\frac{1}{2} F_{z^{\prime} z^{\prime}} L-\frac{1}{2}\left(F_{y^{\prime} z^{\prime}}-G_{z^{\prime} z^{\prime}}\right) M-\frac{1}{2} G_{y^{\prime} z^{\prime}} N .
\end{aligned}
$$

The relations (8.8), (8.9) must be adjoined to (7.5), or $\mathfrak{S}$, so giving the first extended system $\mathfrak{S}_{1}$, which is seen to be arranged in orthonomic form.

We have next to form the passivity conditions of $\mathfrak{\Im}_{1}$.

First we apply $d / d x$ to (8.8) and (8.9). We express all such quantities as $d / d x$ of $L_{z}, M_{y}, L_{y^{\prime}}$, etc., in the manner previously described and then eliminate all principal derivatives, taking due account of the defining conditions of Case I:

$$
A=0, \quad B=0, \quad C=0 .
$$

Then the resulting relation among parametric derivatives is found to disappear identically. Thus, these particular passivity conditions are satisfied, and no new equations need be adjoined on their account.

There are two further passivity conditions of $\mathfrak{S}_{1}$ to consider, represented by

$$
\frac{\partial L_{z}}{\partial z^{\prime}}=\frac{\partial L_{z^{\prime}}}{\partial z}, \quad \frac{\partial N_{y}}{\partial y^{\prime}}=\frac{\partial N_{y^{\prime}}}{\partial y} .
$$

Upon calculation, these two conditions turn out to be the same, namely:

$$
\begin{aligned}
M_{y z^{\prime}}=M_{z y^{\prime}} & +\frac{1}{2} F_{z^{\prime}} M_{y^{\prime} y^{\prime}}-\frac{1}{2}\left(F_{y^{\prime}}-G_{z^{\prime}}\right) M_{y^{\prime} z^{\prime}}-\frac{1}{2} G_{y^{\prime}} M_{z^{\prime} z^{\prime}} \\
& +\frac{1}{2} F_{z^{\prime} z^{\prime}} L_{y^{\prime}}+\frac{1}{2} G_{z^{\prime} z^{\prime}} M_{y^{\prime}}-\frac{1}{2} F_{y^{\prime} y^{\prime}} M_{z^{\prime}}-\frac{1}{2} G_{y^{\prime} y^{\prime}} N_{z^{\prime}} \\
& +\frac{1}{2} F_{y^{\prime} z^{\prime} z^{\prime}} L-\frac{1}{2}\left(F_{y^{\prime} y^{\prime} z^{\prime}}-G_{y^{\prime} z^{\prime} z^{\prime}}\right) M-\frac{1}{2} G_{y^{\prime} y^{\prime} z^{\prime}} N .
\end{aligned}
$$

The relation (8.10) among parametric derivatives of $\mathfrak{S}_{1}$ must be adjoined to that system to form a second extended system $\widetilde{\varsigma}_{2}$.

The system $\widetilde{S}_{2}$ is subject to just one passivity test, namely that in which we take $d / d x$ of (8.10) and then substitute for $d / d x$ of $M_{y z^{\prime}}, M_{z y^{\prime}}, M_{y^{\prime} y^{\prime}}$, etc., the linear differential expressions of second order obtainable in the manner previously described-after which we eliminate all principal derivatives. When this is done, with due account taken of $A=0, B=0, C=0$, it is found after long calculations that the result is an identity: $0=0$.

Therefore: in Case I, the second extension $\mathfrak{S}_{2}$ of the differential system $\subseteq$ is passive.

$\mathfrak{S}_{2}$, we recall, consists of $(7.5)$-except $A L+B M+C N=0$, which disappears identically-(8.8), (8.9), (8.10).

$\mathfrak{S}_{2}$ is also orthonomic, as we see by reference to the table of cotes (8.6); therefore, by the Riquier theory, $\mathfrak{S}_{2}$ is completely integrable.

A complete set of parametric derivatives of $\widetilde{S}_{2}$ with their corresponding multipliers is seen, by the rules of the theory of differential systems $\left({ }^{22}\right)$, to be

(22) Cf. J. M. Thomas, loc. cit., $\$ \S 75,76$. 


$$
\begin{gathered}
L: \text { multipliers } y, y^{\prime} ; \\
N: \text { multipliers } z, z^{\prime} ; \\
M: \text { multipliers } y, z, y^{\prime} ; \\
M_{z^{\prime}}: \text { multipliers } z, y^{\prime}, z^{\prime} .
\end{gathered}
$$

This means that every parametric derivative of $\mathfrak{S}_{2}$ can be expressed in one and only one of the forms: $\partial^{r+s} / \partial y^{r} \partial y^{\prime s}$ of $L, \partial^{r+s} / \partial z^{r} \partial z^{\prime s}$ of $N, \partial^{r+s+t} / \partial y^{r} \partial z^{s} \partial y^{\prime t}$ of $M, \partial^{r+s+t} / \partial z^{r} \partial y^{\prime s} \partial z^{\prime t}$ of $M_{z^{\prime}}$.

It follows that a solution of $\Im_{2}$ (equivalent to $\mathfrak{S}$ ) exists and is uniquely determined if we assign as arbitrary analytic functions, regular about $(0,0,0,0,0)$ (which, without essential loss of generality, we may take as initial values of $\left.x, y, z, y^{\prime}, z^{\prime}\right)$, the following:

$$
\begin{aligned}
f\left(y, y^{\prime}\right) & =L\left(0, y, 0, y^{\prime}, 0\right), \\
g\left(z, z^{\prime}\right) & =N\left(0,0, z, 0, z^{\prime}\right), \\
h\left(y, z, y^{\prime}\right) & =M\left(0, y, z, y^{\prime}, 0\right), \\
k\left(z, y^{\prime}, z^{\prime}\right) & =M_{z^{\prime}}\left(0,0, z, y^{\prime}, z^{\prime}\right) .
\end{aligned}
$$

It is only necessary to provide that

$$
f(0,0) g(0,0)-h(0,0,0)^{2} \neq 0
$$

in order to take care of the inequation $L N-M^{2} \neq 0$.

Instead of $(0,0,0,0,0)$, we may use any system of initial values $\left(x_{0}, y_{0}\right.$, $\left.z_{0}, y_{0}^{\prime}, z_{0}^{\prime}\right)$ about which functions $F, G$ are regular analytic.

Thus, the general solution of $\subseteq$ in Case I depends on two arbitrary functions of three arguments, and two arbitrary functions of two arguments. As will be seen by comparison with the later cases, this is the highest possible degree of generality of the variation problem corresponding to a given curve family as extremals. $\$ 3$.

The results of this section have been summarized in Theorem I, stated in

Example. Case I is illustrated in a fairly general way by the example

$$
y^{\prime \prime}=f\left(z^{\prime}\right), \quad z^{\prime \prime}=0,
$$

$f$ denoting an arbitrary function; this is easily verified to obey the requisite conditions (8.2). For $f\left(z^{\prime}\right)=0$, we have the particular case of the straight lines, already treated in the paper by G. Hamel cited in $\$ 2$.

We may use this example to show also the advantages that may be derived from a knowledge of the finite equations of the given curve family.

The differential equations (8.14) may be integrated, giving

$$
y=\frac{1}{2} f\left(a_{1}\right) x^{2}+a_{3} x+a_{4}, \quad z=a_{1} x+a_{2},
$$


which expresses the given curve family in terms of four parameters: $a_{1}, a_{2}, a_{3}, a_{4}$. By differentiation of (8.15),

$$
y^{\prime}=f\left(a_{1}\right) x+a_{3}, \quad z^{\prime}=a_{1} .
$$

The system (8.15), (8.16) may be solved for $a_{1}, a_{2}, a_{3}, a_{4}$ :

$$
\begin{aligned}
& a_{1}=z^{\prime}, \\
& a_{2}=z-z^{\prime} x, \\
& a_{3}=y^{\prime}-f\left(z^{\prime}\right) x, \\
& a_{4}=y-y^{\prime} x+\frac{1}{2} f\left(z^{\prime}\right) x^{2} ;
\end{aligned}
$$

and we may transform from $x, y, z, y^{\prime}, z^{\prime}$ as independent variables to $x, a_{1}, a_{2}, a_{3}, a_{4} . d / d x$ then denotes an operation of differentiation as to $x$ while $a_{1}, a_{2}, a_{3}, a_{4}$ are kept constant, that is, partial differentiation as to $x$ of a function of $x, a_{1}, a_{2}, a_{3}, a_{4}$.

The first three equations of (7.5) now become

$$
\frac{d L}{d x}=0, \quad \frac{d M}{d x}=-\frac{1}{2} f^{\prime}\left(a_{1}\right) L, \quad \frac{d N}{d x}=-f^{\prime}\left(a_{1}\right) M .
$$

This is a linear system of ordinary differential equations, whose general solution is readily expressed by means of three parameters $\lambda, \mu, \nu$, arbitrary functions of $a_{1}, a_{2}, a_{3}, a_{4}$, as follows:

$$
\begin{aligned}
L & =\lambda, \\
M & =-\frac{1}{2} f^{\prime}\left(a_{1}\right) \lambda x+\mu, \\
N & =\frac{1}{4} f^{\prime 2}\left(a_{1}\right) \lambda x^{2}-f^{\prime}\left(a_{1}\right) \mu x+\nu .
\end{aligned}
$$

We substitute these values in the other two relations of (7.5): $L_{z^{\prime}}=M_{y^{\prime}}$, $N_{y^{\prime}}=M_{z^{\prime}}$, and transform the independent variables to $x, a_{1}, a_{2}, a_{3}, a_{4}$ according to (8.17); in this way we arrive at the following differential system, where the subscripts $1,2,3,4$ denote differentiation as to $a_{1}, a_{2}, a_{3}, a_{4}$, respectively:

$$
\begin{aligned}
& \lambda_{1}=\mu_{3}, \\
& \lambda_{2}=\mu_{4}-\frac{1}{2} f^{\prime}\left(a_{1}\right) \lambda_{3}, \\
& \nu_{3}=\mu_{1}, \\
& \nu_{4}=\mu_{2}+\frac{1}{2} f^{\prime}\left(a_{1}\right) \mu_{3}+\frac{1}{2} f^{\prime \prime}\left(a_{1}\right) \lambda .
\end{aligned}
$$

This replaces completely the original differential system (7.5).

The system (8.20) has two passivity conditions: $\lambda_{12}=\lambda_{21}, \nu_{34}=\nu_{43}$; but on calculation these give the same relation among parametric derivatives of (8.20) (as was to be expected from our previous general theory; cf. the statement preceding (8.10)), namely:

$$
\mu_{14}=\mu_{23}+\frac{1}{2} f^{\prime}\left(a_{1}\right) \mu_{33}+\frac{1}{2} f^{\prime \prime}\left(a_{1}\right) \lambda_{3} .
$$


Since there is no other equation in the system (8.20), (8.21) whose first member is a derivative of $\mu$, there is no further passivity test to be made, and the system in question is passive.

In the case of the straight lines, $f\left(a_{1}\right)=0$, and the passive system to be integrated reduces to

$$
\lambda_{1}=\mu_{3}, \quad \lambda_{2}=\mu_{4} ; \quad \nu_{3}=\mu_{1}, \quad \nu_{4}=\mu_{2} ; \quad \mu_{14}=\mu_{23} .
$$

A general solution of the last equation, in $\mu$ alone, is uniquely determined if $\mu\left(a_{1}, a_{2}, a_{3}, 0\right), \mu_{4}\left(0, a_{2}, a_{3}, a_{4}\right)$ are assigned arbitrarily. Then the differentials $\mu_{3} d a_{1}+\mu_{4} d a_{2}$ and $\mu_{1} d a_{3}+\mu_{2} d a_{4}$ are exact, and give by their integration:

$$
\begin{aligned}
& \lambda=\int\left(\mu_{3} d a_{1}+\mu_{4} d a_{2}\right)+g\left(a_{3}, a_{4}\right), \\
& \nu=\int\left(\mu_{1} d a_{3}+\mu_{2} d a_{4}\right)+h\left(a_{1}, a_{2}\right),
\end{aligned}
$$

where the functions $g, h$ are arbitrary. By (8.19) with $f\left(a_{1}\right)=0,(L, M, N)$ $=(\lambda, \mu, \nu)$, where, by (8.17), we may transform back to $x, y, z, y^{\prime}, z^{\prime}$ as independent variables. Then $\phi$ can be found by the procedure of $\S 5$.

9. Case II. The critical cone. Case II, the most complicated and varied in its results and treatment, is that of rank 1 of the matrix $\Delta$, expressed by

$$
\left\|\begin{array}{lll}
A & B & C \\
A_{1} & B_{1} & C_{1}
\end{array}\right\|=0, \quad\|A B C \quad\| \neq 0 .
$$

These conditions imply the existence of a factor $r\left(x, y, z, y^{\prime}, z^{\prime}\right)$ such that

$$
A_{1}=r A, \quad B_{1}=r B, \quad C_{1}=r C .
$$

Then by the recursion formulas (7.9), we have, with a common factor $s$, $A_{2}=s A, B_{2}=s B, C_{2}=s C$, so that (9.1) indeed expresses rank 1 of the matrix $\Delta$.

Let us consider the equation

$$
L N-M^{2}=0,
$$

whose negation constitutes the basic inequation of the system (7.5) or $\widetilde{S}$. If $L, M, N$ are interpreted as cartesian coordinates in an auxiliary 3 -space, this equation represents a quadric cone with vertex at the origin. This cone, which will play a very important part in our theory, will be referred to as the "critical cone" $K$.

It is evident that an arbitrary point on the critical cone is represented in terms of parameters $\rho, \xi$ by

$$
L=\rho \xi^{2}, \quad M=\rho \xi, \quad N=\rho .
$$


We may also say that these equations represent any vector from the origin along an element of the cone; $\xi$ fixes the element and $\rho$ determines the position of the end-point of the vector on that element.

The linear algebraic equation $\left(7.5_{4}\right)$ of the system $\subseteq$ :

$$
A L+B M+C N=0
$$

- not disappearing identically because of the second condition (9.1)-represents a plane $\mathcal{P}$ in the space $(L, M, N)$ passing through the vertex of the critical cone. A definite position of this plane corresponds to any given values of $\left(x, y, z, y^{\prime}, z^{\prime}\right)$ considered as parameters.

The intersection of the plane $P$ with the critical cone $K$ is determined by substituting (9.4) in (9.5), which leads to the quadratic equation

$$
A \xi^{2}+B \xi+C=0 \text {. }
$$

The two values of $\xi$ furnished by this equation determine the two elements in which the plane $\mathcal{P}$ intersects the critical cone. These elements may be real and distinct, conjugate imaginary, or real and coincident.

We shall denote by $\lambda, \mu$ the two roots of the fundamental quadratic equation $(9.6)\left({ }^{23}\right)$; so that we have

$$
\begin{array}{cc}
A \lambda^{2}+B \lambda+C=0, & A \mu^{2}+B \mu+C=0 \\
\lambda=\frac{-B+\left(B^{2}-4 A C\right)^{1 / 2}}{2 A}, & \mu=\frac{-B-\left(B^{2}-4 A C\right)^{1 / 2}}{2 A} \\
\lambda+\mu=-B / A, & \lambda \mu=C / A .
\end{array}
$$

It is of fundamental importance for the subsequent theory to know whether these roots $\lambda, \mu$-known functions of $x, y, z, y^{\prime}, z^{\prime}$ determined by the given curve family - are distinct or coincident, that is, whether the plane $\mathcal{P}$ intersects the critical cone in two distinct elements (real or conjugate imaginary) or, on the other hand, is tangent to this cone along an element.

We therefore divide the following discussion into two cases accordingly:

Case IIa. $B^{2}-4 A C \neq 0, \lambda \neq \mu$;

Case IIb. $B^{2}-4 A C=0, \lambda=\mu$.

10. Case IIa. The plane $P$ intersects the critical cone $K$ in two distinct elements. Let $e_{1}, e_{2}$ denote the two distinct elements of intersection; then these may be considered as coordinate axes in the plane $P$. According to

(23) If $A=0$, one of $\lambda, \mu$ is infinite; and if $A=0, B=0$, both $\lambda$ and $\mu$ are infinite. These difficulties may be met, provided $C \neq 0$, by interchanging the $y$ and $z$ coordinates, which also interchanges $F$ and $G$, and will be seen by (7.6) to have the effect of interchanging $A$ and $-C$, while converting $B$ into $-B$. There remains only the case $A=0, C=0, B \neq 0$ (if also $B=0$, we are in Case I). By the defining conditions (9.1) of Case II, this implies $A_{1}=0, C_{1}=0$, and, by reference to (7.6), (7.9): $F_{z^{\prime}}=0, F_{z}=0, G_{y^{\prime}}=0, G_{y}=0$. We thus have precisely the example (11.5), which is given separate treatment later on. 
(9.4), arbitrary vectors from the origin along $e_{1}, e_{2}$ respectively are represented by

$$
\begin{array}{lll}
L=\rho \lambda^{2}, & M=\rho \lambda, & N=\rho ; \\
L=\sigma \mu^{2}, & M=\sigma \mu, & N=\sigma ;
\end{array}
$$

and any vector from the origin lying in the plane $P$ is representable as the sum of two such vectors:

$$
L=\rho \lambda^{2}+\sigma \mu^{2}, \quad M=\rho \lambda+\sigma \mu, \quad N=\rho+\sigma .
$$

Here $\rho, \sigma$ may be regarded as the oblique cartesian coordinates (real or conjugate imaginary) of any point $(L, M, N)$ in the plane $\mathcal{P}$, referred to the vectors $\left(\lambda^{2}, \lambda, 1\right),\left(\mu^{2}, \mu, 1\right)$ along the elements $e_{1}, e_{2}$ respectively as unit vectors.

Our next step is to make the substitution (10.1) in the equations of the system $\subseteq$ or (7.5), so deriving a differential system in the two unknown functions $\rho\left(x, y, z, y^{\prime}, z^{\prime}\right), \sigma\left(x, y, z, y^{\prime}, z^{\prime}\right)$ that will be fundamental in the treatment of Case IIa. Before proceeding to do this, we establish the following relations:

$$
\begin{aligned}
& \frac{d \lambda}{d x}=\frac{1}{2} F_{z^{\prime}} \lambda^{2}-\frac{1}{2}\left(F_{y^{\prime}}-G_{z^{\prime}}\right) \lambda-\frac{1}{2} G_{y^{\prime}}, \\
& \frac{d \mu}{d x}=\frac{1}{2} F_{z^{\prime}} \mu^{2}-\frac{1}{2}\left(F_{y^{\prime}}-G_{z^{\prime}}\right) \mu-\frac{1}{2} G_{y^{\prime}}
\end{aligned}
$$

Their proof is as follows. By differentiation of (9.7),

$$
(2 A \lambda+B) \frac{d \lambda}{d x}+\left(\lambda^{2} \frac{d A}{d x}+\lambda \frac{d B}{d x}+\frac{d C}{d x}\right)=0 .
$$

By (9.2) and the formulas (7.9),

$$
\begin{aligned}
& \frac{d A}{d x}=\left(F_{y^{\prime}}+r\right) A+\frac{1}{2} F_{z^{\prime}} B, \\
& \frac{d B}{d x}=G_{y^{\prime}} A+\left(\frac{1}{2} F_{y^{\prime}}+\frac{1}{2} G_{z^{\prime}}+r\right) B+F_{z^{\prime}} C, \\
& \frac{d C}{d x}=\frac{1}{2} G_{y^{\prime}} B+\left(G_{z^{\prime}}+r\right) C .
\end{aligned}
$$

Substituting (10.4) in (10.3), we get, with account taken of (9.7):

$$
\begin{aligned}
(2 A \lambda+B) \frac{d \lambda}{d x} & +\frac{1}{2} F_{y^{\prime}} \lambda(2 A \lambda+B)+\frac{1}{2} G_{y^{\prime}}(2 A \lambda+B) \\
& +\frac{1}{2} G_{z^{\prime}}(B \lambda+2 C)+\frac{1}{2} F_{z^{\prime}} \lambda(B \lambda+C)=0 .
\end{aligned}
$$

Now by (9.8). 


$$
2 A \lambda+B=\left(B^{2}-4 A C\right)^{1 / 2}
$$

and by $(9.7,8)$,

$$
B \lambda+2 C=-\lambda(2 A \lambda+B)=-\lambda\left(B^{2}-4 A C\right)^{1 / 2} .
$$

Substituting in (10.5) and cancelling the factor $\left(B^{2}-4 A C\right)^{1 / 2} \neq 0$, we obtain (10.2), as was desired.

Let us then write (10.1) in the first three equations of (7.5); these become, with the help of (10.2):

$$
\begin{array}{r}
\lambda^{2}\left\{\frac{d \rho}{d x}+\left(F_{z^{\prime}} \lambda+G_{z^{\prime}}\right) \rho\right\}+\mu^{2}\left\{\frac{d \sigma}{d x}+\left(F_{z^{\prime}} \mu+G_{z^{\prime}}\right) \sigma\right\}=0, \\
\lambda\left\{\frac{d \rho}{d x}+\left(F_{z^{\prime}} \lambda+G_{z^{\prime}}\right) \rho\right\}+\mu\left\{\frac{d \sigma}{d x}+\left(F_{z^{\prime}} \mu+G_{z^{\prime}}\right) \sigma\right\}=0, \\
\left\{\frac{d \rho}{d x}+\left(F_{z^{\prime}} \lambda+G_{z^{\prime}}\right) \rho\right\}+\left\{\frac{d \sigma}{d x}+\left(F_{z^{\prime}} \mu+G_{z^{\prime}}\right) \sigma\right\}=0 .
\end{array}
$$

Since $\lambda-\mu \neq 0$, these equations obviously imply the zero value of each of the two brackets, that is:

$$
\frac{d \rho}{d x}=-\left(F_{z^{\prime}} \lambda+G_{z^{\prime}}\right) \rho, \quad \frac{d \sigma}{d x}=-\left(F_{z^{\prime}} \mu+G_{z^{\prime}}\right) \sigma .
$$

The fourth equation, $A L+B M+C N=0$, of (7.5) disappears identically after the substitution (10.1).

The remaining equations of $(7.5)$,

$$
L_{z^{\prime}}=M_{y^{\prime}}, \quad N_{y^{\prime}}=M_{z^{\prime}},
$$

give, by the substitution (10.1), two relations, which may be solved for $\rho_{y^{\prime}}$, $\sigma_{y^{\prime}} ;$ we thus obtain

$$
\rho_{y^{\prime}}=\lambda \rho_{z^{\prime}}+\left(\lambda_{z^{\prime}}+\alpha\right) \rho+\beta \sigma, \quad \sigma_{y^{\prime}}=\mu \sigma_{z^{\prime}}+\left(\mu_{z^{\prime}}-\beta\right) \sigma-\alpha \rho,
$$

where

$$
\alpha=\frac{\lambda \lambda_{z^{\prime}}-\lambda_{y^{\prime}}}{\lambda-\mu}, \quad \beta=\frac{\mu \mu_{z^{\prime}}-\mu_{y^{\prime}}}{\lambda-\mu} .
$$

The inequation $L N-M^{2} \neq 0$ of the system $\subseteq$ means that the point $(L, M, N)$ shall not lie upon the critical cone; according to the remarks connected with (10.1), this is expressed by the requirement that both

$$
\rho \neq 0, \quad \sigma \neq 0 .
$$

The same is also seen from the formula: $L N-M^{2}=\rho \sigma(\lambda-\mu)^{2}$, following from (10.1) - since $\lambda-\mu \neq 0$. 
The fundamental differential system $\mathfrak{I}$ of Case IIa consists of the four equations (10.7), (10.8) and the inequations (10.10).

The vanishing or nonvanishing of the quantities $\alpha, \beta$ defined by (10.9) is of fundamental importance in the study of the differential system $\mathfrak{T}$; accordingly, we make the following division of Case IIa into subcases:

Case IIa1. The "separated case":

$$
\lambda \lambda_{z^{\prime}}-\lambda_{y^{\prime}}=0, \quad \mu \mu_{z^{\prime}}-\mu_{y^{\prime}}=0 .
$$

Case IIa2. The "semi-separated case":

$$
\lambda \lambda_{z^{\prime}}-\lambda_{y^{\prime}}=0, \quad \mu \mu_{z^{\prime}}-\mu_{y^{\prime}} \neq 0 .
$$

Case IIa3. The "non-separated case":

$$
\lambda \lambda_{z^{\prime}}-\lambda_{y^{\prime}} \neq 0, \quad \mu \mu_{z^{\prime}}-\mu_{y^{\prime}} \neq 0 .
$$

Since $\lambda, \mu$ are defined as the roots of $A \xi^{2}+B \xi+C=0$, the preceding conditions can also be expressed in terms of $A, B, C$; we find, by calculations that the reader may easily supply, for the separated case:

$$
\begin{aligned}
& I \equiv B\left(B A_{z^{\prime}}-A B_{z^{\prime}}\right)+A\left(B A_{y^{\prime}}-A B_{y^{\prime}}\right)+A\left(A C_{z^{\prime}}-C A_{z^{\prime}}\right)=0, \\
& J \equiv B\left(B C_{y^{\prime}}-C B_{y^{\prime}}\right)+C\left(B C_{z^{\prime}}-C B_{z^{\prime}}\right)+C\left(C A_{y^{\prime}}-A C_{y^{\prime}}\right)=0 ;
\end{aligned}
$$

for the semi-separated case:

$$
C^{3} I^{2}+\left(B^{3}-3 A B C\right) I J+A^{3} J^{2}=0, \quad \text { with either } I \neq 0 \text {, or } J \neq 0 ;
$$

for the non-separated case:

$$
C^{3} I^{2}+\left(B^{3}-3 A B C\right) I J+A^{3} J^{2} \neq 0 .
$$

The names we have given to our three subcases refer to the fact that, first, when $\alpha=0, \beta=0$, the system $\mathfrak{T}$ separates into two parts involving respectively $\rho$ alone and $\sigma$ alone:

$$
\frac{d \rho}{d x}=-\left(F_{z^{\prime}} \lambda+G_{z^{\prime}}\right) \rho, \quad \rho_{y^{\prime}}=\lambda \rho_{z^{\prime}}+\lambda_{z^{\prime}} \rho
$$

and

$$
\frac{d \sigma}{d x}=-\left(F_{z^{\prime}} \mu+G_{z^{\prime}}\right) \sigma, \quad \sigma_{y^{\prime}}=\mu \sigma_{z^{\prime}}+\mu_{z^{\prime}} \sigma
$$

Second, when $\alpha=0, \beta \neq 0$, we have a system of two equations involving $\sigma$ alone, namely:

$$
\frac{d \sigma}{d x}=-\left(F_{z^{\prime}} \mu+G_{z^{\prime}}\right) \sigma, \quad \sigma_{y^{\prime}}=\mu \sigma_{z^{\prime}}+\dot{(}\left(\mu_{z^{\prime}}-\beta\right) \sigma,
$$


combined with a mixed system where $\sigma$ and $\rho$ occur together:

$$
\frac{d \rho}{d x}=-\left(F_{z^{\prime}} \lambda+G_{z^{\prime}}\right) \rho, \quad \rho_{y^{\prime}}=\lambda \rho_{z^{\prime}}+\lambda_{z^{\prime}} \rho+\beta \sigma ;
$$

hence the name "semi-separated."

Finally, when $\alpha \neq 0, \beta \neq 0$, we have the original system (10.7), (10.8) with no separation into partial systems one or both of which involve only one of the unknowns $\rho, \sigma$; hence "non-separated."

Throughout the discussion of Case IIa, in all its parts, we shall adopt the following system of cotes:

\begin{tabular}{l|l|l|l|l|l|l}
\hline \hline$x$ & $y$ & $y^{\prime}$ & $z$ & $z^{\prime}$ & $\rho$ & $\sigma$ \\
\hline 1 & 1 & 1 & 1 & 1 & 0 & 0 \\
1 & 0 & 0 & 0 & 0 & 0 & 0 \\
0 & 1 & 0 & 0 & 0 & 0 & 0 \\
0 & 0 & 1 & 0 & 0 & 0 & 0 \\
0 & 0 & 0 & 1 & 0 & 0 & 0 \\
0 & 0 & 0 & 0 & 1 & 0 & 0 \\
0 & 0 & 0 & 0 & 0 & 2 & 1 \\
\hline
\end{tabular}

This is equivalent to arranging the derivatives of $\rho, \sigma$ first as to their total order, then as to their order in $x$, in $y$, in $y^{\prime}$, in $z$, in $z^{\prime}$, then as to the unknown function in the order $\rho, \sigma$.

We shall solve all our equations for the derivative of highest cote which appears effectively, or-even if this is not done explicitly-our equations may always be thought of as solved for this highest derivative. Thus all the differential systems to be treated will have the orthonomic form essential for application of the Riquier theory. Sometimes, however, a form in which some additional terms are transposed will be employed as technically more desirable. This will be done on those occasions when we shall form the passivity condition of two equations by building the alternant of corresponding linear differential operators of first order. Advantageous here will be the simplification which arises from the fact that the alternant is again of the first order, all second order terms which are apparently introduced cancelling. This device of alternants will not interfere, however, with the applicability of the Riquier theory of orthonomic systems, in which it produces only inessential modifications.

11. Case IIa1. The separated case. Here we have to solve the system consisting of (10.17), (10.18), (10.10), under the conditions (10.11).

Each of the partial systems comes under the classic theory of simultaneous linear partial differential equations of first order in a single unknown func- 
tion $\left({ }^{24}\right)$. Forming the alternant of the two equations of $(10.17)$, we find

$$
\rho_{y}=\lambda \rho_{z}+H \rho_{z^{\prime}}+P_{1} \rho,
$$

where

$$
\begin{aligned}
& H=\frac{d \lambda}{d x}=\frac{1}{2} F_{z^{\prime}} \lambda^{2}-\frac{1}{2}\left(F_{y^{\prime}}-G_{z^{\prime}}\right) \lambda-\frac{1}{2} G_{y^{\prime}}, \quad \text { by }(10.2), \\
& P_{1}=\lambda_{z}+H_{z^{\prime}} .
\end{aligned}
$$

If we next form the alternant of (11.1) with (10.171), this can be calculated to disappear identically as a consequence of the equations already in the system.

The alternant of (11.1) with $\left(10.16_{2}\right)$ can be calculated to be

$$
\left(\lambda \lambda_{z^{\prime}}-\lambda_{y^{\prime}}\right) \rho_{z}+U \rho_{z^{\prime}}+X_{1} \rho=0,
$$

where

$$
\begin{aligned}
U & =\frac{d}{d x}\left(\lambda \lambda_{z^{\prime}}-\lambda_{y^{\prime}}\right)+\left(F_{y^{\prime}}-\lambda F_{z^{\prime}}\right)\left(\lambda \lambda_{z^{\prime}}-\lambda_{y^{\prime}}\right), \\
X_{1} & =U_{z^{\prime}}+\frac{\partial}{\partial z}\left(\lambda \lambda_{z^{\prime}}-\lambda_{y^{\prime}}\right) .
\end{aligned}
$$

But since $\lambda \lambda_{z^{\prime}}-\lambda_{y^{\prime}}=0$, equation (11.2) disappears identically; therefore (10.17), (11.1) constitute a complete system for $\rho$.

Similarly, we find the following complete system for $\sigma:(10.18)$ together with

$$
\sigma_{\nu}=\mu \sigma_{z}+K \sigma_{z^{\prime}}+Q_{1} \sigma
$$

where

$$
\begin{aligned}
& K=\frac{d \mu}{d x}=\frac{1}{2} F_{z^{\prime}} \mu^{2}-\frac{1}{2}\left(F_{y^{\prime}}-G_{z^{\prime}}\right) \mu-\frac{1}{2} G_{y^{\prime}}, \quad \text { by }(10.2), \\
& Q_{1}=\mu_{z}+K_{z^{\prime}} .
\end{aligned}
$$

Indeed, the alternant of (11.3) with $\left(10.18_{2}\right)$ is

$$
\left(\mu \mu_{z^{\prime}}-\mu_{y^{\prime}}\right) \sigma_{z}+V \sigma_{z^{\prime}}+Y_{1} \sigma=0,
$$

where

$$
\begin{aligned}
V & =\frac{d}{d x}\left(\mu \mu_{z^{\prime}}-\mu_{y^{\prime}}\right)+\left(F_{y^{\prime}}-\mu F_{z^{\prime}}\right)\left(\mu \mu_{z^{\prime}}-\mu_{y^{\prime}}\right), \\
Y_{1} & =V_{z^{\prime}}+\frac{\partial}{\partial z}\left(\mu \mu_{z^{\prime}}-\mu_{y^{\prime}}\right)
\end{aligned}
$$

${ }^{(24)}$ For a presentation of this theory, see Goursat-Hedrick, Differential Equations (vol. 2, part 2 of $A$ Course in Mathematical Analysis, Boston, 1917), p. 265 et seq. 
so that the equation (11.4) disappears identically because of $\mu \mu_{z^{\prime}}-\mu_{y^{\prime}}=0$.

Since each function $\rho, \sigma$ involves five independent arguments and is a solution of a complete system of three equations, the corresponding variation problem has the generality $\infty^{2 f(2)}\left({ }^{25}\right)$.

Example.

$$
y^{\prime \prime}=F\left(x, y, y^{\prime}\right), \quad z^{\prime \prime}=G\left(x, z, z^{\prime}\right), \quad B \neq 0 .
$$

As we shall see, our inverse problem separates into one in the $x y$-plane and one in the $x z$-plane.

It is evident by (7.6) and (7.9) that $A=0, C=0, A_{1}=0, C_{1}=0$, so that by (9.1) we are indeed in Case II.

Since by hypothesis, $B \neq 0$, the formula $A L+B M+C N=0$ gives $M=0$ as the equation of the fundamental plane $\mathcal{P}$. We may regard $L, N$ instead of $\rho, \sigma$ as the coordinates in this plane. The system (7.5) becomes in the present case:

$$
\begin{array}{ll}
\frac{d L}{d x}+F_{y^{\prime}} L=0, & L_{z^{\prime}}=0 ; \\
\frac{d N}{d x}+G_{z^{\prime}} N=0, & N_{y^{\prime}}=0 ;
\end{array}
$$

which is clearly of the separated type. Forming alternants, we find

$$
L_{z}=0, \quad N_{y}=0 .
$$

By (11.8), and the second equations of (11.6), (11.7),

$$
L=L\left(x, y, y^{\prime}\right), \quad N=N\left(x, z, z^{\prime}\right) .
$$

Equations $\left(11.6_{1}\right),\left(11.7_{1}\right)$ may then be written

$$
\begin{aligned}
& {\left[\frac{\partial}{\partial x}+y^{\prime} \frac{\partial}{\partial y}+F\left(x, y, y^{\prime}\right) \frac{\partial}{\partial y^{\prime}}\right] L=-F_{y^{\prime}} L,} \\
& {\left[\frac{\partial}{\partial x}+z^{\prime} \frac{\partial}{\partial z}+G\left(x, z, z^{\prime}\right) \frac{\partial}{\partial z^{\prime}}\right] N=-G_{z^{\prime}} N .}
\end{aligned}
$$

Since $L=\phi_{y^{\prime} y^{\prime}}, M=\phi_{y^{\prime} z^{\prime}}, N=\phi_{z^{\prime} z^{\prime}}$, the condition $M=0$ together with (11.9) implies for $\phi$ the separated form

$$
\phi=\theta\left(x, y, y^{\prime}\right)+\psi\left(x, z, z^{\prime}\right),
$$

within an additive expression of the linear form

$$
\alpha(x, y, z)+\beta(x, y, z) y^{\prime}+\gamma(x, y, z) z^{\prime} .
$$

Clearly,

(25) Cf. Goursat-Hedrick, loc. cit., p. 270. 


$$
L=\theta_{y^{\prime} y^{\prime}}, \quad N=\psi_{z^{\prime} z^{\prime}} .
$$

The formulas (11.10), (11.13) are precisely those which are met with in solving the following inverse problems in the $x y$ - and $x z$-planes respectively $\left({ }^{26}\right)$ :

(a) find $\int \theta\left(x, y, y^{\prime}\right) d x=\min$, given the extremals $y^{\prime \prime}=F\left(x, y, y^{\prime}\right)$;

(b) find $\int \psi\left(x, z, z^{\prime}\right) d x=$ min, given the extremals $z^{\prime \prime}=G\left(x, z, z^{\prime}\right)$.

According to (11.11), the solutions of these planar problems are to be added to give the solution of the space problem. The undetermined additive expression (11.12) can be proved, as usual, to be an arbitrary total derivative, $\nu_{x}+\nu_{y} y^{\prime}+\nu_{z} z^{\prime}$ where $\nu=\nu(x, y, z)$.

12. Case IIa2. The semi-separated case. Here we have to deal with the differential system (10.19), (10.20), (10.10), under the conditions (10.12).

The alternant of the two equations of $(10.19)$ is

$$
\sigma_{y}=\mu \sigma_{z}+K \sigma_{z^{\prime}}+Q \sigma,
$$

while the integrability condition of the two equations of (10.20), arising from formation of the alternant

$$
\frac{d}{d x}\left(\frac{\partial}{\partial y^{\prime}}-\lambda \frac{\partial}{\partial z^{\prime}}\right)-\left(\frac{\partial}{\partial y^{\prime}}-\lambda \frac{\partial}{\partial z^{\prime}}\right) \frac{d}{d x}
$$

applied to $\rho$, is

$$
\rho_{y}=\lambda \rho_{z}+H \rho_{z^{\prime}}+P_{1} \rho+S \sigma .
$$

Here

$$
\begin{aligned}
H & =\frac{d \lambda}{d x}=\frac{1}{2} F_{z^{\prime}} \lambda^{2}-\frac{1}{2}\left(F_{y^{\prime}}-G_{z^{\prime}}\right) \lambda-\frac{1}{2} G_{y^{\prime}}, \\
K & =\frac{d \mu}{d x}=\frac{1}{2} F_{z^{\prime}} \mu^{2}-\frac{1}{2}\left(F_{y^{\prime}}-G_{z^{\prime}}\right) \mu-\frac{1}{2} G_{y^{\prime}} \\
P_{1} & =\lambda_{z}+H_{z^{\prime}}, \\
Q & =\mu_{z}+K_{z^{\prime}}-S \\
S & =-\frac{d \beta}{d x}-\left(F_{y^{\prime}}-\mu F_{z^{\prime}}\right) \beta .
\end{aligned}
$$

The alternant of $\left(10.19_{1}\right)$ and (12.1) can be calculated to disappear identically in virtue of the equations of the system, and the same is true of $\left(10.20_{1}\right)$ and (12.2)

The alternant of $\left(10.19_{2}\right)$ and (12.1) is

$$
\left(\mu \mu_{z^{\prime}}-\mu_{y^{\prime}}\right) \sigma_{z}+V \sigma_{z^{\prime}}+Y_{2} \sigma=0 .
$$

where

${ }^{(28)}$ Cf. Bolza. loc. cit. (see Footnote 4). 


$$
\begin{aligned}
V & =\frac{d}{d x}\left(\mu \mu_{z^{\prime}}-\mu_{y^{\prime}}\right)+\left(F_{y^{\prime}}-\mu F_{z^{\prime}}\right)\left(\mu \mu_{z^{\prime}}-\mu_{y^{\prime}}\right) \\
Y_{2} & =V_{z^{\prime}}+\frac{\partial}{\partial z}\left(\mu \mu_{z^{\prime}}-\mu_{y^{\prime}}\right)+S_{y^{\prime}}-\mu S_{z^{\prime}}-\beta_{y}+\mu \beta_{z}+K \beta_{z^{\prime}}
\end{aligned}
$$

It is remarkable that precisely the same equation (12.4) is also the integrability condition of $\left(10.20_{2}\right)$ and (12.2), as found by forming the alternant of the linear differential operators

and

$$
\frac{\partial}{\partial y^{\prime}}-\lambda \frac{\partial}{\partial z^{\prime}}
$$

$$
\frac{\partial}{\partial y}-\lambda \frac{\partial}{\partial z}-H \frac{\partial}{\partial z^{\prime}}
$$

applied to $\rho$. It follows that if any solution $\sigma$ of the system (10.19), (12.1), (12.4) is substituted in (10.20), (12.2), this becomes a complete system for $\rho$.

All depends then on solving the system (10.19), (12.1), (12.4) for $\sigma$. It is evident that the formation of further alternants-(12.4) with $\left(10.20_{1}\right)$, with $\left(10.20_{2}\right)$, with (12.1)-gives new equations of the form

$$
\cdot \sigma_{z^{\prime}}+\cdot \sigma=0
$$

where the dots represent calculable known coefficients. There are just two of this type, since the one arising from $\left(10.20_{1}\right)$ and (12.4) can be shown to disappear identically.

If the two equations (12.6) are independent as algebraic linear equations in $\sigma_{z^{\prime}}, \sigma$ (determinant $\neq 0$ ), then $\sigma=0$, in contradiction with our prescribed inequation $\sigma \neq 0$. The given curve family is then nonextremal.

If the two equations (12.6) disappear identically (each coefficient equal to zero), then $\left(10.20_{1}\right),\left(10.20_{2}\right),(12.1),(12.4)$ constitute a complete system of four equations for $\sigma$, with a solution, therefore, of the generality $\infty^{1 f(1)}$. Any such solution substituted in the system for $\rho$ renders this a complete system containing three equations, whose solution therefore has the generality $\infty^{1 f(2)}$. The given curve family is consequently of extremal type and the corresponding integrand $\phi$ has the generality $\infty^{1 f(2)+1 f(1)}$.

Finally, let the two equations (12.6) amount to exactly one independent equation (coefficient-matrix of rank one). If the coefficient of $\sigma_{z^{\prime}}$ in this equation is zero, then that of $\sigma$ cannot be zero (because of the rank one); it follows that $\sigma=0$, in contradiction with the stipulated inequation $\sigma \neq 0$, and we have a nonextremal family. On the other hand, if the coefficient of $\sigma_{z^{\prime}}$ is not equal to zero, then we have a system of five equations of the form

$$
\sigma_{x}=\cdot \sigma, \quad \sigma_{y}=\cdot \sigma, \quad \sigma_{z}=\cdot \sigma, \quad \sigma_{y^{\prime}}=\cdot \sigma, \quad \sigma_{z^{\prime}}=\cdot \sigma,
$$


where the dots represent known coefficients. The solvability of this system for $\sigma(\neq 0)$ depends on the exactness of the differential

$$
\cdot d x+\cdot d y+\cdot d z+\cdot d y^{\prime}+\cdot d z^{\prime},
$$

where the dots are respectively the coefficients in (12.7). The solution, if existent, is unique up to a constant factor (which may be ignored according to the remarks associated with (3.7)). The generality of the corresponding variation problem is $\infty^{1 f(2)}$, arising out of that degree of generality in the system for $\rho$.

The results of this section have been summarized in Theorem III of $\$ 3$.

Example. Catenaries. The $y$-axis in $x y z$-space being taken vertical, the differential equations

$$
y^{\prime \prime}=\frac{1+y^{\prime 2}+z^{\prime 2}}{y}, \quad z^{\prime \prime}=0
$$

represent all vertical catenaries the directrix of each of which coincides with the trace of its plane on the horizontal $x z$-plane.

Here we calculate by (7.6), (7.9):

$$
\begin{array}{ccc}
A=-\frac{4 y^{\prime} z^{\prime}}{y^{2}}, & B=-\frac{4\left(1+z^{\prime 2}\right)}{y^{2}}, & C=0 ; \\
A_{1}=\frac{12 y^{\prime 2} z^{\prime}}{y^{3}}, & B_{1}=\frac{12 y^{\prime}\left(1+z^{\prime 2}\right)}{y^{3}}, & C_{1}=0 .
\end{array}
$$

Thus the defining conditions (9.1) of Case II are verified.

The fundamental quadratic equation $(9.6)$ is here

$$
y^{\prime} z^{\prime} \xi^{2}+\left(1+z^{\prime 2}\right) \xi=0,
$$

whose roots are

$$
\lambda=0, \quad \mu=-\frac{1+z^{\prime 2}}{y^{\prime} z^{\prime}} .
$$

The system $(10.19,20)$ can in this case be calculated to be

$$
\begin{gathered}
\frac{d \rho}{d x}=0, \quad \rho_{y^{\prime}}=-\frac{1}{y^{\prime} z^{\prime 2}} \sigma ; \\
\frac{d \sigma}{d x}=2 \frac{1+z^{\prime 2}}{y y^{\prime}} \sigma, \quad \sigma_{y^{\prime}}=-\frac{1+z^{\prime 2}}{y^{\prime} z^{\prime}} \sigma_{z^{\prime}}+\frac{2-z^{\prime 2}}{y^{\prime} z^{\prime 2}} \sigma ;
\end{gathered}
$$

with the prescribed inequations $\rho \neq 0, \sigma \neq 0$.

Forming the alternant of the two equations of (12.12), we get

$$
\rho_{y}=\frac{1+y^{\prime 2}+z^{\prime 2}}{y y^{\prime 2} z^{\prime 2}} \sigma .
$$


If we form the alternant of the two equations of (12.13), we obtain the equation

$$
\sigma_{y}=-\frac{1+z^{\prime 2}}{y^{\prime} z^{\prime}} \sigma_{z}+K \sigma_{z^{\prime}}+Q \sigma,
$$

where

$$
\begin{aligned}
& K=\frac{\left(1+z^{\prime 2}\right)\left(1+y^{\prime 2}+z^{\prime 2}\right)}{y y^{\prime 2} z^{\prime}} \\
& Q=\frac{-2\left(1+y^{\prime 2}\right)+\left(1+y^{\prime 2}\right) z^{\prime 2}+3 z^{\prime 4}}{y y^{\prime 2} z^{\prime 2}} .
\end{aligned}
$$

The alternant of (12.15) with the first of (12.13) disappears identically in virtue of the equations of the system. The alternant of (12.15) with the second of (12.13) is simply

$$
\sigma_{z}=0 .
$$

(12.13), (12.15), (12.16) can be verified to constitute a complete system for $\sigma$, all further alternants being linear consequences of the equations of the system.

The integrability conditions of (12.12), (12.14) are satisfied in virtue of the complete system for $\sigma$. Accordingly, all our equations form a completely integrable (passive orthonomic) system in $\rho, \sigma$, the generality of whose solution is seen to be $\infty 1 f(2)+1 f(1)$.

13. Case IIa3. The non-separated case. It may first be remarked that the class of curve families $(F, G)$ coming under this case is quite extensive. For the conditions for Case IIa3 are that the two partial differential equations of third order in $F, G$ :

$$
\frac{A_{1}}{A}=\frac{B_{1}}{B}=\frac{C_{1}}{C}
$$

have a solution which obeys at least one of the inequations $A \neq 0, B \neq 0, C \neq 0$ and also the inequation (10.16). Now, according to standard existence theorems (Cauchy-Kovalevsky), the differential system (13.1), consisting of two equations for two unknown functions, has a solution involving a number of arbitrary functions in its initial data. It is easy to adjust these arbitrary functions so as to obey all the specified inequations.

We proceed, then, to deal with the differential system of Case IIa3, which consists of (10.7), (10.8) and the inequations (10.10), under the conditions (10.13).

Forming the integrability conditions of $\left(10.7_{1}\right)$ with $\left(10.8_{1}\right)$ and of $\left(10.7_{2}\right)$ with $\left(10.8_{2}\right)$, we find 


$$
\begin{aligned}
& \rho_{y}=\lambda \rho_{z}+H \rho_{z^{\prime}}+P \rho+S \sigma, \\
& \sigma_{y}=\mu \sigma_{z}+K \sigma_{z^{\prime}}+Q \sigma+R \rho,
\end{aligned}
$$

where

$$
\begin{aligned}
& H=\frac{d \lambda}{d x}=\frac{1}{2} F_{z^{\prime}} \lambda^{2}-\frac{1}{2}\left(F_{y^{\prime}}-G_{z^{\prime}}\right) \lambda-\frac{1}{2} G_{y^{\prime}} \\
& K=\frac{d \mu}{d x}=\frac{1}{2} F_{z^{\prime}} \mu^{2}-\frac{1}{2}\left(F_{y^{\prime}}-G_{z^{\prime}}\right) \mu-\frac{1}{2} G_{y^{\prime}} \\
& R=\frac{d \alpha}{d x}+\left(F_{y^{\prime}}-\lambda F_{z^{\prime}}\right) \alpha \\
& S=-\frac{d \beta}{d x}-\left(F_{y^{\prime}}-\mu F_{z^{\prime}}\right) \beta \\
& P=\lambda_{z}+H_{z^{\prime}}-R \\
& Q=\mu_{z}+K_{z^{\prime}}-S .
\end{aligned}
$$

The integrability condition of $\left(10.7_{1}\right)$ with (13.21) disappears identically in virtue of the equations of the system, and the same is true of $\left(10.7_{2}\right)$ and $\left(13.2_{2}\right)$.

On the other hand, the integrability condition of $\left(10.8_{1}\right)$ with $\left(13.2_{1}\right)$ is found after calculation to be

$$
\left(\lambda \lambda_{z^{\prime}}-\lambda_{y^{\prime}}\right) \rho_{z}+U \rho_{z^{\prime}}+X \rho=\left(\mu \mu_{z^{\prime}}-\mu_{y^{\prime}}\right) \sigma_{z}+V \sigma_{z^{\prime}}+Y \sigma
$$

where

$$
\begin{aligned}
U & =\lambda_{y}-\lambda \lambda_{z}-H \lambda_{z^{\prime}}+\lambda H_{z^{\prime}}-H_{y^{\prime}} \\
& =\frac{d}{d x}\left(\lambda \lambda_{z^{\prime}}-\lambda_{y^{\prime}}\right)+\left(F_{y^{\prime}}-\lambda F_{z^{\prime}}\right)\left(\lambda \lambda_{z^{\prime}}-\lambda_{y^{\prime}}\right) \\
& =(H-K) \alpha+(\lambda-\mu) R \\
V & =\mu_{y}-\mu \mu_{z}-K \mu_{z^{\prime}}+\mu K_{z^{\prime}}-K_{y^{\prime}} \\
(13.5) \quad= & \frac{d}{d x}\left(\mu \mu_{z^{\prime}}-\mu_{y^{\prime}}\right)+\left(F_{y^{\prime}}-\mu F_{z^{\prime}}\right)\left(\mu \mu_{z^{\prime}}-\mu_{y^{\prime}}\right) \\
& =(H-K) \beta-(\lambda-\mu) S ; \\
X & =\beta R+\alpha S+U_{z^{\prime}}+\frac{\partial}{\partial z}\left(\lambda \lambda_{z^{\prime}}-\lambda_{y^{\prime}}\right)+R_{y^{\prime}}-\lambda R_{z^{\prime}}+\alpha_{y}-\lambda \alpha_{z}-H \alpha_{z^{\prime}}, \\
Y & =-\beta R-\alpha S+V_{z^{\prime}}+\frac{\partial}{\partial z}\left(\mu \mu_{z^{\prime}}-\mu_{y^{\prime}}\right)+S_{y^{\prime}}-\mu S_{z^{\prime}}-\beta_{y}+\mu \beta_{z}+K \beta_{z^{\prime}} .
\end{aligned}
$$


If we interchange $\lambda$ with $\mu$, the effect, as seen by our defining formulas, is to interchange $\alpha$ and $-\beta, H$ and $K, R$ and $S, P$ and $Q, U$ and $V, X$ and $Y$. If at the same time we interchange $\rho$ and $\sigma$, the equation (13.4) is seen to go over into itself. This is at the basis of the important fact that the same equation (13.4) is also obtained as the integrability condition of $\left(10.8_{2}\right)$ with $\left(13.2_{2}\right)$.

Let us introduce a third unknown function $\tau$ to denote the common value of the two members of (13.4):

$$
\begin{aligned}
& \tau=\left(\lambda \lambda_{z^{\prime}}-\lambda_{y^{\prime}}\right) \rho_{z}+U \rho_{z^{\prime}}+X \rho, \\
& \tau=\left(\mu \mu_{z^{\prime}}-\mu_{y^{\prime}}\right) \sigma_{z}+V \sigma_{z^{\prime}}+Y \sigma .
\end{aligned}
$$

Since, by hypothesis, $\lambda \lambda_{z^{\prime}}-\lambda_{y^{\prime}} \neq 0, \mu \mu_{z^{\prime}}-\mu_{y^{\prime}} \neq 0$, we can then give our differential system the form:

$$
\begin{aligned}
\frac{d \rho}{d x} & =\cdot \rho, \\
\rho_{y^{\prime}} & =\cdot \rho_{z^{\prime}}+\cdot \rho+\cdot \sigma, \\
\rho_{y} & =\cdot \rho_{z^{\prime}}+\cdot \rho+\cdot \sigma+\cdot \tau, \\
\rho_{z} & =\cdot \rho_{z^{\prime}}+\cdot \rho+\cdot \sigma+\cdot \tau ; \\
\frac{d \sigma}{d x} & =\cdot \sigma, \\
\sigma_{y^{\prime}} & =\cdot \sigma_{z^{\prime}}+\cdot \rho+\cdot \sigma+\cdot \tau, \\
\sigma_{y} & =\cdot \sigma_{z^{\prime}}+\cdot \rho+\cdot \sigma+\cdot \tau, \\
\sigma_{z} & =\cdot \sigma_{z^{\prime}}+\cdot \rho+\cdot \sigma+\cdot \tau ;
\end{aligned}
$$

where the dots represent calculable known coefficients. If now we form the new integrability conditions of this system $\left({ }^{27}\right)$, we find that the resulting equations are solvable for the first partial derivatives of $\tau$ (in which circumstance the requirement $\lambda-\mu \neq 0$ plays a part); indeed, we get a result of the form

$$
\begin{aligned}
\frac{d \tau}{d x} & =\cdot \rho+\cdot \sigma+\cdot \tau\left({ }^{28}\right), \\
\tau_{y} & =\cdot \rho_{z^{\prime}}+\cdot \sigma_{z^{\prime}}+\cdot \rho+\cdot \sigma+\cdot \tau, \\
\tau_{z} & =\cdot \rho_{z^{\prime}}+\cdot \sigma_{z^{\prime}}+\cdot \rho+\cdot \sigma+\cdot \tau, \\
\tau_{y^{\prime}} & =\cdot \rho_{z^{\prime}}+\cdot \sigma_{z^{\prime}}+\cdot \rho+\cdot \sigma+\cdot \tau, \\
\tau_{z^{\prime}} & =\cdot \rho_{z^{\prime}}+\cdot \sigma_{z^{\prime}}+\cdot \rho+\cdot \sigma+\cdot \tau .
\end{aligned}
$$

(27) We may use the formulas (13.6) as convenient for this purpose, building alternants of the linear operators in the second members of (13.6) with the linear operators occurring in (10.7), (10.8), (13.2).

(28) Two expressions are obtained for $d \tau / d x$, but these can be calculated to be equal in virtue of our other formulas. The coefficient of $\tau$ in the first equation of $(13.8)$ is $-\left(F_{y^{\prime}}+G_{z^{\prime}}\right)$. 
The differential system we now have to consider is $(13.7),(13.8)$ in the three unknown functions $\rho, \sigma, \tau$.

Referring to (10.21), we give $\tau$ a last cote of 3 and all other cotes 0 , thus ranking $\tau$ higher than $\rho, \sigma$. The differential system in question then has the orthonomic form.

Forming the integrability conditions of (13.8) (such as $\partial \tau_{y} / \partial z=\partial \tau_{z} / \partial y$ ), we obtain, after reduction with the help of (13.7), a number of equations of the form

$$
\cdot \rho_{z^{\prime} z^{\prime}}+\cdot \sigma_{z^{\prime} z^{\prime}}+\cdot \rho_{z^{\prime}}+\cdot \sigma_{z^{\prime}}+\cdot \rho+\cdot \sigma+\cdot \tau=0 .
$$

If all these equations disappear identically, the system (13.7), (13.8) is passive, and has a solution corresponding to arbitrarily assigned values of $\rho\left(0,0,0,0, z^{\prime}\right), \sigma\left(0,0,0,0, z^{\prime}\right):$ generality $\infty 2 f(1)$.

Laying this case aside, we may find it possible to eliminate some of the partial derivatives from (13.9) by linear combination of these equations. If the rank of the whole system is $r$, while the rank of the coefficients of $\rho_{z^{\prime} z^{\prime}}$, $\sigma_{z^{\prime} z^{\prime}}, \rho_{z^{\prime}}, \sigma_{z^{\prime}}$ is $r-p$ ( $p$ necessarily $\geqq 0$ ), it will be possible to infer in this way exactly $p$ independent relations of the form

$$
\cdot \rho+\cdot \sigma+\cdot \tau=0 \text {. }
$$

In case $p=3,(13.10)$ implies $\rho=0, \sigma=0, \tau=0$, in contradiction with the prescribed inequations $\tau \neq 0, \sigma \neq 0$.

If $p=2$, it is possible to eliminate $\tau$ and derive a relation of the form $\sigma=\cdot \rho($ or $\rho=\cdot \sigma)$. By substitution in the original differential system (10.7), (10.8), this takes the form

$$
\rho_{x}=\cdot \rho, \quad \rho_{y}=\cdot \rho, \quad \rho_{z}=\cdot \rho, \quad \rho_{y^{\prime}}=\cdot \rho, \quad \rho_{z^{\prime}}=\cdot \rho,
$$

together with a condition of the form $(\cdot) \rho=0$-all the dots representing calculable known coefficients. The integrability conditions of (13.11) are of the form $(\cdot) \rho=0$, so that it is necessary and sufficient for the existence of a solution $\rho \neq 0$ that all the coefficients $(\cdot)$ be equal to zero. If this is the case, $\rho$ is determined uniquely up to a constant factor.

In case only one independent equation of the type (13.10) can be obtained, we may suppose it solvable for $\tau$ :

$$
\tau=\cdot \rho+\cdot \sigma
$$

otherwise we find ourselves in the situation just discussed. Substituting (13.12) in (13.7), we have a system in $\rho, \sigma$, whose integrability conditions are those which result by substituting (13.12) in (13.18). The first equation of (13.8) gives in this way a relation of the form $\cdot \rho+\cdot \sigma=0$ which-if it does not disappear identically, or imply $\rho=0$ or $\sigma=0$-puts us in the preceding case. If this relation, $\cdot \rho+\cdot \sigma=0$, does disappear identically, the substitution of (13.12) in the other relations of (13.8) may be used, with (13.7), to give in- 
tegrability conditions of the form

$$
\cdot \rho_{z^{\prime}}+\cdot \sigma_{z^{\prime}}+\cdot \rho+\cdot \sigma=0 .
$$

If (13.13) disappears identically, we have in (13.7) (with $\tau$ replaced by its value (13.12)) a passive system in $\rho, \sigma$.

If, by linear combination of the equations of (13.13), we can eliminate $\rho_{z^{\prime}}, \sigma_{z^{\prime}}$, that is, infer a relation of the form $\cdot \rho+\cdot \sigma=0$, we are in a previously discussed case.

If we cannot do this, it must be that the rank of the whole system (13.13) is the same as the rank of the coefficients of $\rho_{z^{\prime}}, \sigma_{z^{\prime}}$. This common rank may be 2 or 1 , since we have disposed of the case of rank zero of the system.

If the rank is 2 , we can solve for $\rho_{z^{\prime}}, \sigma_{z^{\prime}}$ :

$$
\rho_{z^{\prime}}=\cdot \rho+\cdot \sigma, \quad \sigma_{z^{\prime}}=\cdot \rho+\cdot \sigma .
$$

Now by substituting (13.12), (13.14) in (13.7), every first partial derivative of $\rho, \sigma$ is expressed in the form $\cdot \rho+\cdot \sigma$ :

$$
\begin{aligned}
\frac{d \rho}{d x} & =\cdot \rho, & \frac{d \sigma}{d x} & =\cdot \sigma, \\
\rho_{y} & =\cdot \rho+\cdot \sigma, & \sigma_{y} & =\cdot \rho+\cdot \sigma, \\
\rho_{z} & =\cdot \rho+\cdot \sigma, & \sigma_{z} & =\cdot \rho+\cdot \sigma, \\
\rho_{y^{\prime}} & =\cdot \rho+\cdot \sigma, & \sigma_{y^{\prime}} & =\cdot \rho+\cdot \sigma, \\
\rho_{z^{\prime}} & =\cdot \rho+\cdot \sigma ; & \sigma_{z^{\prime}} & =\cdot \rho+\cdot \sigma .
\end{aligned}
$$

This is a system of a well-known type, easily amenable to treatment by standard methods $\left({ }^{29}\right)$. It is obvious that here all the integrability conditions are of the form $\cdot \rho+\cdot \sigma=0$. If there are two such independent conditions, we infer $\rho=0, \sigma=0$, in violation of the prescribed inequations $\rho \neq 0, \sigma \neq 0$. If there is only one such condition, then, first, neither coefficient may be zero, otherwise we again have contradiction with either $\rho \neq 0$ or $\sigma \neq 0$. Assuming neither coefficient zero, our system has a solution for $\rho, \sigma$, which is essentially unique (within a constant factor). Finally, if all the integrability conditions $\cdot \rho+\cdot \sigma=0$ disappear identically, (13.15) is a completely integrable system, with a solution involving linearly two arbitrary constants (only one of which is essential).

If the rank mentioned in the second preceding paragraph is 1 , then (13.13) consists of just one equation solvable for either $\rho_{z^{\prime}}$ or $\sigma_{z^{\prime}}$, say the former:

$$
\rho_{z^{\prime}}=\cdot \sigma_{z^{\prime}}+\cdot \rho+\cdot \sigma .
$$

Substituting this, as well as (13.12), in (13.7), we have a differential system in $\rho, \sigma$, whose integrability conditions are a set of equations of the form

${ }^{(29)}$ Cf. Eisenhart, An Introduction to Differential Geometry, Princeton, 1940, p. 114. 


$$
\cdot \sigma_{z^{\prime} z^{\prime}}+\cdot \sigma_{z^{\prime}}+\cdot \rho+\cdot \sigma=0 .
$$

If, by elimination, we can derive from these equations a relation of the form $\sigma_{z^{\prime}}=\cdot \rho+\cdot \sigma$, or $\cdot \rho+\cdot \sigma=0$ (non-identically vanishing), then we are evidently in previously discussed cases $((13.15)$ or (13.11)). If not, it must be that $(13.15 \mathrm{~b})$ contains just one independent equation, and that solvable in the form

$$
\sigma_{z^{\prime} z^{\prime}}=\cdot \sigma_{z^{\prime}}+\cdot \rho+\cdot \sigma .
$$

Adjoining this to the equations already in our possession, we have a differential system in which the only parametric derivatives are $\sigma_{z^{\prime}}, \rho, \sigma$. All further integrability conditions are therefore of the form

$$
\cdot \sigma_{z^{\prime}}+\cdot \rho+\cdot \sigma=0 \text {. }
$$

Either all of these disappear identically, in which case we have a passive system, or else we derive at least one relation of the form $\sigma_{z^{\prime}}=\cdot \rho+\cdot \sigma$, or $\cdot \rho+\cdot \sigma=0$-wherewith our investigation is reduced, as before, to previously discussed cases.

We have now disposed of the case where even one relation of the form (13.10) can be inferred linearly from (13.9).

If this cannot be done, it may still be possible to eliminate $\rho_{z^{\prime} z^{\prime}}, \sigma_{z^{\prime} z^{\prime}}$ from (13.9) and obtain relations of the form

$$
\cdot \rho_{z^{\prime}}+\cdot \sigma_{z^{\prime}}+\cdot \rho+\cdot \sigma+\cdot \tau=0 .
$$

Let $r$ denote the rank of (13.9) and $r-q(q \geqq 0)$ the rank of the coefficients of $\rho_{z^{\prime} z^{\prime}}, \sigma_{z^{\prime} z^{\prime}}$; then exactly $q$ independent relations of the form (13.16) can be inferred by linear combination of (13.9). We are thus supposing $q \geqq 1$-otherwise no relations of the type (13.16) are derivable by the elimination process mentioned.

If $q$ were as large as 3, we could eliminate $\rho_{z^{\prime}}, \sigma_{z^{\prime}}$ from (13.16) and obtain a relation of the form (13.10); but we have supposed this not to be the case.

If $q=2$, then we can solve the two relations (13.16) for $\rho_{z^{\prime}}, \sigma_{z^{\prime}}$ (otherwise it would be possible to eliminate these, contrary to hypothesis) and get:

$$
\rho_{z^{\prime}}=\cdot \rho+\cdot \sigma+\cdot \tau, \quad \sigma_{z^{\prime}}=\cdot \rho+\cdot \sigma+\cdot \tau .
$$

Substituting in (13.7), (13.8), we have a system where every first partial derivative of $\rho, \sigma, \tau$ is expressed in the form $\cdot \rho+\cdot \sigma+\cdot \tau$, that is, a system of the standard type (13.15) with three unknown functions instead of two. All the integrability conditions of this system are of the form $\cdot \rho+\cdot \sigma+\cdot \tau=0$, so that we have an easy discussion based on the number of such independent conditions $(0,1$, or 2$)$ not in contradiction with $\rho \neq 0, \sigma \neq 0$.

If $q=1$, we can solve the single equation (13.16) for $\rho_{z^{\prime}}$ or $\sigma_{z^{\prime}}$, say:

$$
\rho_{z^{\prime}}=\cdot \sigma_{z^{\prime}}+\cdot \rho+\cdot \sigma+\cdot \tau \text {. }
$$


The integrability conditions of this with the first group of (13.7) are of the form

$$
\cdot \sigma_{z^{\prime} z^{\prime}}+\cdot \sigma_{z^{\prime}}+\cdot \rho+\cdot \sigma+\cdot \tau=0 .
$$

If we can eliminate $\sigma_{z^{\prime} z^{\prime}}$ from these equations, we clearly have cases already discussed. Otherwise, (13.19) must consist of just one equation and this solvable for $\sigma_{z^{\prime} z^{\prime}}$ :

$$
\sigma_{z^{\prime} z^{\prime}}=\cdot \sigma_{z^{\prime}}+\cdot \rho+\cdot \sigma+\cdot \tau .
$$

After adjunction of this to the preceding equations of our system, the only partial derivatives which remain are the four: $\sigma_{z^{\prime}}, \rho, \sigma, \tau$, so that all further integrability conditions must involve only these in linear homogeneous form. Accordingly, with the formation of at most four additional integrability conditions we come to a decision as to the solvability of our system (in conformity with $\rho \neq 0, \sigma \neq 0$ ) and as to the number of arbitrary constants (at most four) in the solution.

Now there remains for the system (13.9) only the case where no relations can be derived by elimination of $\rho_{z^{\prime} z^{\prime}}, \sigma_{z^{\prime} z^{\prime}}$. In this case, the rank of the entire system is equal to the rank of the coefficients of $\rho_{z^{\prime} z^{\prime}}, \sigma_{z^{\prime} z^{\prime}}$. The possible values of this common rank $s$ are 2,1 , since $s=0$ has already been laid aside as a case of passivity of the system.

If $s=2$, we can solve (13.19) in the form

$$
\begin{aligned}
& \rho_{z^{\prime} z^{\prime}}=\cdot \rho_{z^{\prime}}+\cdot \sigma_{z^{\prime}}+\cdot \rho+\cdot \sigma+\cdot \tau, \\
& \sigma_{z^{\prime} z^{\prime}}=\cdot \rho_{z^{\prime}}+\cdot \sigma_{z^{\prime}}+\cdot \rho+\cdot \sigma+\cdot \tau .
\end{aligned}
$$

Then the five quantities appearing in the second member are the only remaining parametric derivatives. All further integrability conditions have the form of a linear homogeneous relation in these quantities, so that with the formation of at most five of these relations (supposed independent) we come to a decision.

Finally, let $s=1$. Then (13.9) consists of exactly one relation, which is solvable for either $\rho_{z^{\prime} z^{\prime}}$ or $\sigma_{z^{\prime} z^{\prime}}\left({ }^{30}\right)$, say:

$$
\rho_{z^{\prime} z^{\prime}}=\cdot \sigma_{z^{\prime} z^{\prime}}+\cdot \rho_{z^{\prime}}+\cdot \sigma_{z^{\prime}}+\cdot \rho+\cdot \sigma+\cdot \tau .
$$

The integrability conditions of this with the system (13.7), first part, are of the form

$$
\cdot \sigma_{z^{\prime} z^{\prime} z^{\prime}}+\cdot \sigma_{z^{\prime} z^{\prime}}+\cdot \rho_{z^{\prime}}+\cdot \sigma_{z^{\prime}}+\cdot \rho+\cdot \sigma+\cdot \tau=0 .
$$

If we can eliminate $\sigma_{z^{\prime} z^{\prime} z^{\prime}}$, we are in previously discussed cases. The only alternative is that (13.23) contain just one equation, and this solvable for $\sigma_{z^{\prime} z^{\prime} z^{\prime}}$ :

$$
\sigma_{z^{\prime} z^{\prime} z^{\prime}}=\cdot \sigma_{z^{\prime} z^{\prime}}+\cdot \rho_{z^{\prime}}+\cdot \sigma_{z^{\prime}}+\cdot \rho+\cdot \sigma+\cdot \tau .
$$

$\left.{ }^{(30}\right)$ Otherwise, we are obviously in the case associated with (13.16). 
After adjunction of this equation, the only parametric derivatives remaining in our differential system are the six which appear in the second member of (13.24). All further integrability conditions are linear homogeneous relations in these quantities, so that after formation of at most six such (independent) relations, we arrive at a decision as to the consistency of our differential system and the degree of generality of its solution.

We may conclude by emphasizing that the determination of the explicit value of any of the coefficients symbolized by dots throughout the preceding discussion requires only differentiations, multiplications, and additions.

We may also call attention to the fact that throughout the discussion of Case IIa, even in the most unfavorable eventualities, the only derivatives of an order higher than the first which have occurred are $\rho_{z^{\prime} z^{\prime}}, \sigma_{z^{\prime} z^{\prime}}, \sigma_{z^{\prime} z^{\prime} z^{\prime}}$.

14. Case IIb. The plane $P$ is tangent to the critical cone. In the case indicated by the title of this section, let the element of contact $e$ be the one containing the point $\left(\lambda^{2}, \lambda, 1\right)$. As $\lambda$ varies, the locus of this point is a conic, whose tangent vector is $(2 \lambda, 1,0)$. The plane $P$ determined by these two vectors is the one which is tangent to the cone $K$ along the element $e$. The equation of this plane being $A L+B M+C N=0, \lambda$ is evidently determined as the double root of the quadratic

$$
A \lambda^{2}+B \lambda+C=0, \quad\left(B^{2}-4 A C=0\right) .
$$

The coordinates $(L, M, N)$ of an arbitrary point in the plane $P$ can be written as a linear combination of the two vectors previously mentioned with arbitrary multipliers $\rho, \sigma$ :

$$
L=\lambda^{2} \rho+2 \lambda \rho, \quad M=\lambda \rho+\sigma, \quad N=\rho .
$$

Here $\lambda$ is given as a known function of $x, y, z, y^{\prime}, z^{\prime}$ by (14.1); indeed,

$$
\lambda=-\frac{B}{2 A}=-\frac{2 C}{B},
$$

while $\rho, \sigma$ are unknown functions of $x, y, z, y^{\prime}, z^{\prime}$.

Substituting (14.2) in the original differential system $\subseteq$, or (7.5), we obtain, after reduction, the following differential system for $\rho, \sigma$ :

$$
\begin{aligned}
& \frac{d \rho}{d x}=-\left(F_{z^{\prime}} \lambda+G_{z^{\prime}}\right) \rho-F_{z^{\prime}} \sigma, \\
& \frac{d \sigma}{d x}=-\frac{1}{2}\left(F_{y^{\prime}}+G_{z^{\prime}}\right) \sigma \\
& \rho_{y^{\prime}}=\lambda \rho_{z^{\prime}}+\lambda_{z^{\prime}} \rho+\sigma_{z^{\prime}}, \\
& \sigma_{y^{\prime}}=\lambda \sigma_{z^{\prime}}+(\mathrm{I}) \rho+2 \lambda_{z^{\prime}} \sigma,
\end{aligned}
$$

where 


$$
\text { (I) }=\lambda \lambda_{z^{\prime}}-\lambda_{y^{\prime}}
$$

We must also adjoin the inequation

$$
\sigma \neq 0 \text {, }
$$

in order to prevent the point $(L, M, N)$ from lying upon the critical cone.

Our problem in Case IIb is to discuss the solvability for $\rho, \sigma$ of the differential system consisting of the four equations (14.4) and the inequation (14.6).

To this end, we begin by forming the alternant of $\left(14.4_{1}\right),\left(14.4_{3}\right)$, and also the alternant of $\left(14.4_{2}\right),\left(14.4_{4}\right)$. We find:

$$
\begin{aligned}
& \rho_{y}=\lambda \rho_{z}+(\mathrm{II}) \rho_{z^{\prime}}+(\mathrm{III}) \rho+\sigma_{z}+(\mathrm{IV}) \sigma_{z^{\prime}}+(\mathrm{V}) \sigma \\
& \sigma_{y}=\lambda \sigma_{z}+(\mathrm{II}) \sigma_{z^{\prime}}+(\mathrm{VI}) \sigma+(\mathrm{VII}) \rho
\end{aligned}
$$

where the Roman numerals in parentheses denote known functions whose values are:

$$
\begin{aligned}
(\mathrm{II})= & \frac{1}{2} F_{z^{\prime}} \lambda^{2}-\frac{1}{2}\left(F_{y^{\prime}}-G_{z^{\prime}}\right) \lambda-\frac{1}{2} G_{y^{\prime}} \\
(\mathrm{III})= & \lambda_{z}+(\mathrm{II})_{z^{\prime}} \\
(\mathrm{IV})= & F_{z^{\prime}} \lambda-\frac{1}{2}\left(F_{y^{\prime}}-G_{z^{\prime}}\right) \\
(\mathrm{V})= & (\mathrm{IV})_{z^{\prime}} \\
(\mathrm{VI})= & 2 \lambda_{z}+2 \lambda_{z^{\prime}}(\mathrm{IV})-\lambda(\mathrm{IV})_{z^{\prime}}+(\mathrm{IV})_{y^{\prime}} \\
= & 2 \lambda_{z}+F_{z^{\prime}}\left(\lambda \lambda_{z^{\prime}}+\lambda_{y^{\prime}}\right)-\left(F_{y^{\prime}}-G_{z^{\prime}}\right) \lambda_{z^{\prime}} \\
& -F_{z^{\prime} z^{\prime}} \lambda^{2}+\frac{1}{2}\left(3 F_{y^{\prime} z^{\prime}}-G_{z^{\prime} z^{\prime}}\right) \lambda-\frac{1}{2}\left(F_{y^{\prime} y^{\prime}}-G_{y^{\prime} z^{\prime}}\right) \\
(\mathrm{VII})= & (\mathrm{I})\left(2 F_{z^{\prime}} \lambda-\frac{3}{2} F_{y^{\prime}}+\frac{1}{2} G_{z^{\prime}}\right)-\frac{d(\mathrm{I})}{d x} \\
= & \lambda \lambda_{z}-\lambda_{y}+(\mathrm{II}) \lambda_{z^{\prime}}-\lambda(\mathrm{II})_{z^{\prime}}+(\mathrm{II})_{y^{\prime}}+(\mathrm{I})(\mathrm{IV}) .
\end{aligned}
$$

The equations (14.7) must be adjoined to the original system (14.4). With the same cotes (10.21) that were used in Case IIa, the enlarged system is seen to be in orthonomic form. We proceed to consider this system as to its passivity.

The passivity conditions arising from the equations solved for $d \rho / d x$, $\rho_{y^{\prime}}, \rho_{y}$ are found, after some calculation, to be verified identically. The same is true of the passivity condition arising by comparison of the equations solved for $d \sigma / d x, \sigma_{y}$. However, when we apply to the equations solved for $\sigma_{y^{\prime}}, \sigma_{y}$ the relation $\partial \sigma_{y^{\prime}} / \partial y=\partial \sigma_{y} / \partial y^{\prime}$-or, what is equivalent, when we form the alternant of the linear differential operators on $\sigma$ occurring in the two equationswe obtain the passivity condition

$$
2(\mathrm{I}) \sigma_{z}+\{2(\mathrm{I})(\mathrm{IV})-(\mathrm{VII})\} \sigma_{z^{\prime}}+(\mathrm{VIII}) \rho+(\mathrm{IX}) \sigma=0,
$$

where 


$$
\begin{aligned}
(\mathrm{VIII})= & \lambda(\mathrm{VII})_{z^{\prime}}-(\mathrm{VII})_{y^{\prime}}+(\mathrm{I})_{y}-\lambda(\mathrm{I})_{z}+\lambda_{z^{\prime}}(\mathrm{VII}) \\
& -(\mathrm{II})(\mathrm{I})_{z^{\prime}}+(\mathrm{I})(\mathrm{III})-(\mathrm{I})(\mathrm{VI}), \\
(\mathrm{IX})= & \lambda(\mathrm{VI})_{z^{\prime}}-(\mathrm{VI})_{y^{\prime}}-2 \lambda_{z^{\prime} z^{\prime}}(\mathrm{II})+2 \lambda_{y z^{\prime}}-2 \lambda \lambda_{z z^{\prime}}+(\mathrm{I})(\mathrm{V}) .
\end{aligned}
$$

15. Case IIb1. We may now divide the discussion of the differential system $(14.4,6)$ into two parts as follows:

$$
\begin{array}{ll}
\text { Case IIb1. } & (\mathrm{I}) \equiv \lambda \lambda_{z^{\prime}}-\lambda_{y^{\prime}}=0, \\
\text { Case IIb2. } & \text { (I) } \equiv \lambda \lambda_{z^{\prime}}-\lambda_{y^{\prime}} \neq 0 .
\end{array}
$$

If $(\mathrm{I})=0$, the equations $\left(14.4_{2}\right),\left(14.4_{4}\right)$ involve $\sigma$ alone. Their alternant is $\left(14.7_{2}\right)$, where, by the last formula of $(14.8),(\mathrm{VII})=0$. After adjoining $\left(14.7_{2}\right)$, we form the alternant (14.9). Here, because of $(I)=0$, (VII) $=0$, the coefficients of $\sigma_{z}, \sigma_{z^{\prime}}$ are zero, while also (VIII) $=0$ by (14.10). Hence (14.9) reduces to

$$
\left(\mathrm{IX}^{\prime}\right) \sigma=0,
$$

where $\left(\mathrm{IX}^{\prime}\right)$ denotes (IX) without its last term, this vanishing because of $(\mathrm{I})=0$.

Accordingly, we are led to subdivide Case IIb1 into

$$
\begin{array}{lll}
\text { Case IIb1'. } & (\mathrm{I})=0, & \left(\mathrm{IX}^{\prime}\right)=0 ; \\
\text { Case IIb1'. } & (\mathrm{I})=0, & \left(\mathrm{IX}^{\prime}\right) \neq 0 .
\end{array}
$$

In Case IIb1', the equations (14.42), (14.44), (14.72) form a complete linear differential system of first order in $\sigma$ alone-the passivity condition (14.9), or (15.2), disappearing identically. If any solution $\sigma$ of this system is substituted in the remaining equations of $(14.4,7)$, these form a complete system for $\rho$. Hence a solution of the total system in $\rho, \sigma$ exists, of the generality $\infty^{2 f(2)}$. This result is expressed in Theorem IV of $\$ 3$.

In Case IIb1", the equation (15.2) implies $\sigma=0$, in contradiction with the prescribed inequation $\sigma \neq 0$. Hence, in this case the given family of curves is nonextremal.

Example of Case IIb1'.

$$
y^{\prime \prime}=z, \quad z^{\prime \prime}=0 .
$$

Here by (7.6), (7.9),

$$
\begin{aligned}
& A=-2, \quad B=0, \quad C=0 ; \\
& A_{1}=0, \quad B_{1}=0, \quad C_{1}=0 \text {. }
\end{aligned}
$$

Hence (9.1) is verified, and we are in Case II.

The quadratic (9.6) is here $\lambda^{2}=0$, with the double root $\lambda=0$. Since this obeys $(\mathrm{I})=0$, we have all the conditions of Case IIb1. 
The differential system (14.4) is here

$$
\begin{array}{ll}
\frac{d \rho}{d x}=0, & \frac{d \sigma}{d x}=0, \\
\rho_{y^{\prime}}=\sigma_{z^{\prime}}, & \sigma_{y^{\prime}}=0 .
\end{array}
$$

To this we adjoin the passivity conditions corresponding to (14.7):

$$
\rho_{y}=\sigma_{z}, \quad \sigma_{y}=0 .
$$

The unique passivity condition of this system, corresponding to (14.9), is found to disappear identically, since $\left(\mathrm{IX}^{\prime}\right)=0$; thus $(15.6,7)$ form a completely in tegrable system.

It is profitable here, as in many other cases, to use the finite equations of the extremals as well as their differential equations (15.4). Integrating the latter, we find

$$
\begin{aligned}
y & =\frac{1}{6} a_{1} x^{3}+\frac{1}{2} a_{2} x^{2}+a_{3} x+a_{4}, & z & =a_{1} x+a_{2} ; \\
y^{\prime} & =\frac{1}{2} a_{1} x^{2}+a_{2} x+a_{8}, & z^{\prime} & =a_{1} .
\end{aligned}
$$

These equations may be solved for the four arbitrary constants $a_{1}, a_{2}, a_{3}, a_{4}$ :

$$
\begin{aligned}
& a_{1}=z^{\prime}, \\
& a_{2}=z-z^{\prime} x, \\
& a_{3}=y^{\prime}-x z+\frac{1}{2} z^{\prime} x^{2}, \\
& a_{4}=y-y^{\prime} x+\frac{1}{2} z x^{2}-\frac{1}{6} z^{\prime} x^{3} .
\end{aligned}
$$

The system $(15.6,7)$ may be transformed from $x, y, z, y^{\prime}, z^{\prime}$ as independent variables to $x, a_{1}, a_{2}, a_{3}, a_{4}$; it becomes

$$
\begin{aligned}
\frac{\partial \rho}{\partial x} & =0, \quad \frac{\partial \sigma}{\partial x}=0, \\
\rho_{3}-x \rho_{4} & =\sigma_{1}-x \sigma_{2}+\frac{1}{2} x^{2} \sigma_{3}-\frac{1}{6} x^{3} \sigma_{4}, \\
\rho_{4} & =\sigma_{2}-x \sigma_{3}+\frac{1}{2} x^{2} \sigma_{4}, \\
\sigma_{3}-x \sigma_{4} & =0, \\
\sigma_{4} & =0 .
\end{aligned}
$$

Here the symbol for partial differentiation, $\partial / \partial x$, is used instead of $d / d x$, since now, in differentiation as to $x$, this variable alone changes in value while the other independent variables $a_{1}, a_{2}, a_{3}, a_{4}$ are held fixed. The subscripts $1,2,3,4$ denote differentiation as to the latter variables respectively.

By the first two equations of (15.10), $\rho=\rho\left(a_{1}, a_{2}, a_{3}, a_{4}\right), \sigma=\sigma\left(a_{1}, a_{2}, a_{3}, a_{4}\right)$. The remaining four equations can be simplified as follows, with the disappearance of $x$ : 


$$
\begin{array}{ll}
\rho_{3}=\sigma_{1}, & \rho_{4}=\sigma_{2}, \\
\sigma_{3}=0, & \sigma_{4}=0 .
\end{array}
$$

By the last two,

$$
\sigma=f\left(a_{1}, a_{2}\right),
$$

$f$ being an arbitrary function; and then by the first two:

$$
\rho=a_{3} f_{1}\left(a_{1}, a_{2}\right)+a_{4} f_{2}\left(a_{1}, a_{2}\right)+g\left(a_{1}, a_{2}\right),
$$

where $f_{1} \equiv \partial f / \partial a_{1}, f_{2} \equiv \partial f / \partial a_{2}$, and $g$ is a second arbitrary function.

By (14.2), since $\lambda=0$, we have $L=0, M=\sigma, N=\rho$; hence by $(15.12,13)$ and the substitutions (15.9), we have $L, M, N$ as functions of $x, y, z, y^{\prime}, z^{\prime}$. The integrand $\phi$ can then be found by the method of $\S 5$.

Example of Case IIb1". Let $G=0$; then $C=0$. Let $F$ be such that

$$
B=0, \quad A \neq 0, \quad F_{y^{\prime} y^{\prime} y^{\prime}} \neq 0 .
$$

These conditions are consistent, since the partial differential equation $B=0$ obeyed by $F$, that is,

$$
F_{y^{\prime} y^{\prime}}=\left\{2 F_{y}+\frac{1}{2} F_{y^{\prime}}^{2}-F_{x y^{\prime}}-y^{\prime} F_{y^{\prime} y^{\prime}}-z^{\prime} F_{z y^{\prime}}-G F_{y^{\prime} z^{\prime}}\right\} \div F,
$$

is of the Cauchy normal form, and therefore has a solution corresponding to arbitrarily given analytic functions $f, g$ if we prescribe that

$$
f\left(x, y, z, z^{\prime}\right)=F\left(x, y, z, 0, z^{\prime}\right), \quad g\left(x, y, z, z^{\prime}\right)=F_{y^{\prime}}\left(x, y, z, 0, z^{\prime}\right) .
$$

It is evidently possible to choose these two arbitrary functions so that the inequations in (15.14) are satisfied.

By (7.9) and our stated conditions, we have

$$
B_{1}=0, \quad C_{1}=0 ;
$$

therefore, since also $B=0, C=0, A \neq 0$, we are in Case II. The fundamental quadratic, $A \xi^{2}+B \xi+C=0$, has the double root $\lambda=0$, for which $\lambda \lambda_{z^{\prime}}-\lambda_{y^{\prime}}=0$; we thus have the conditions of Case IIb1.

Further, with reference to (14.8), we have

$$
(\mathrm{I})=0, \quad(\mathrm{II})=0, \quad(\mathrm{VI})=-\frac{1}{2} F_{y^{\prime} y^{\prime}}, \quad(\mathrm{VII})=0 ;
$$

therefore $\left(14.4_{4}\right),\left(14.7_{2}\right)$ become

$$
\sigma_{y^{\prime}}=0, \quad \sigma_{y}=-\frac{1}{2} F_{y^{\prime} y^{\prime}} \sigma .
$$

Taking $\partial / \partial y^{\prime}$ of the second equation and using the first, we get as the analogue of (14.9):

$$
F_{y^{\prime} y^{\prime} y^{\prime}} \sigma=0
$$


consequently, by the stipulation $F_{y^{\prime} y^{\prime} y^{\prime}} \neq 0$ of (15.14), we deduce $\sigma=0$, in contradiction with the prescribed inequation $\sigma \neq 0$.

16. Case IIb2. In this case, where $(I) \neq 0$, the passivity condition (14.9) of the system $(14.4,7)$ is not verified identically, nor does it offer any direct contradiction with the prescribed inequation $\sigma \neq 0$; therefore we adjoin (14.9) and proceed to form further passivity conditions.

With the abbreviation

$$
(\mathrm{X})=2(\mathrm{I})(\mathrm{IV})-(\mathrm{VII})
$$

we rewrite (14.9) as

$$
\left[2(\mathrm{I}) \frac{\partial}{\partial z}+(\mathrm{X}) \frac{\partial}{\partial z^{\prime}}\right] \sigma=-(\mathrm{VIII}) \rho-(\mathrm{IX}) \sigma .
$$

Similarly, we write $\left(14.4_{4}\right)$ in the operational form

$$
\left[\frac{\partial}{\partial y^{\prime}}-\lambda \frac{\partial}{\partial z^{\prime}}\right] \sigma=(\mathrm{I}) \rho+2 \lambda_{z^{\prime}} \sigma .
$$

Forming then the alternant of (16.2), (16.3), and using the other equations of our system, we obtain a new first order equation of the form

$$
(\mathrm{XI}) \sigma_{z}+(\mathrm{XII}) \sigma_{z^{\prime}}=(\mathrm{I})\left[2(\mathrm{I}) \rho_{z}+(\mathrm{X}) \rho_{z^{\prime}}\right]+(\mathrm{XIII}) \rho+(\mathrm{XIV}) \sigma,
$$

where the coefficients represented by Roman numerals can be expressed by explicit formulas in terms of the preceding Roman numerals and their partial derivatives, namely:

$$
\begin{aligned}
(\mathrm{XI})= & -2\left[(\mathrm{I})_{y^{\prime}}-\lambda(\mathrm{I})_{z^{\prime}}\right] \\
(\mathrm{XII})= & -2(\mathrm{I}) \lambda_{z}-(\mathrm{X}) \lambda_{z^{\prime}}-(\mathrm{X})_{y^{\prime}}+\lambda(\mathrm{X})_{z^{\prime}}-(\mathrm{VIII}) \\
(\mathrm{XIII})= & 2(\mathrm{I})(\mathrm{I})_{z}+(\mathrm{X})(\mathrm{I})_{z^{\prime}}+(\mathrm{VIII})_{y^{\prime}}-\lambda(\mathrm{VIII})_{z^{\prime}}-\lambda_{z^{\prime}}(\mathrm{VIII}) \\
& +(\mathrm{I})(\mathrm{IX}) \\
(\mathrm{XIV})= & 2\left[2(\mathrm{I}) \lambda_{z z^{\prime}}+(\mathrm{X}) \lambda_{z_{z^{\prime}}}\right]+(\mathrm{IX})_{y^{\prime}}-\lambda(\mathrm{IX})_{z_{z^{\prime}}} .
\end{aligned}
$$

Similarly, we may write $\left(14.7_{2}\right)$ in the operational form

$$
\left[\frac{\partial}{\partial y}-\lambda \frac{\partial}{\partial z}-(\mathrm{II}) \frac{\partial}{\partial z^{\prime}}\right] \sigma=(\mathrm{VI}) \sigma+(\mathrm{VII}) \rho,
$$

and build the alternant of this with (16.2); we get, after reduction with the use of the other equations of the system:

$$
(\mathrm{XV}) \sigma_{z}+(\mathrm{XVI}) \sigma_{z^{\prime}}=(\mathrm{VII})\left[2(\mathrm{I}) \rho_{z}+(\mathrm{X}) \rho_{z^{\prime}}\right]+(\mathrm{XVII}) \rho+(\mathrm{XVIII}) \sigma,
$$

where 


$$
\begin{aligned}
(\mathrm{XV})= & -\left[2(\mathrm{I}) \frac{\partial}{\partial z}+(\mathrm{X}) \frac{\partial}{\partial z^{\prime}}\right] \lambda \\
& -2\left[\frac{\partial}{\partial y}-\lambda \frac{\partial}{\partial z}-(\mathrm{II}) \frac{\partial}{\partial z^{\prime}}\right](\mathrm{I})-(\mathrm{VIII}), \\
(\mathrm{XVI})= & -\left[2(\mathrm{I}) \frac{\partial}{\partial z}+(\mathrm{X}) \frac{\partial}{\partial z^{\prime}}\right](\mathrm{II}) \\
& -\left[\frac{\partial}{\partial y}-\lambda \frac{\partial}{\partial z}-(\mathrm{II}) \frac{\partial}{\partial z^{\prime}}\right](\mathrm{X})-(\mathrm{IV})(\mathrm{VIII}), \\
(\mathrm{XVII})= & {\left[2(\mathrm{I}) \frac{\partial}{\partial z}+(\mathrm{X}) \frac{\partial}{\partial z^{\prime}}\right](\mathrm{VII}) } \\
& +\left[\frac{\partial}{\partial y}-\lambda \frac{\partial}{\partial z}-(\mathrm{II}) \frac{\partial}{\partial z^{\prime}}\right](\mathrm{X}) \\
& +(\mathrm{III})(\mathrm{VIII})-(\mathrm{VI})(\mathrm{VIII})+(\mathrm{VII})(\mathrm{IX}), \\
(\mathrm{XVIII})= & {\left[2(\mathrm{I}) \frac{\partial}{\partial z}+(\mathrm{X}) \frac{\partial}{\partial z^{\prime}}\right](\mathrm{VI}) } \\
& +\left[\frac{\partial}{\partial y}-\lambda \frac{\partial}{\partial z}-(\mathrm{II}) \frac{\partial}{\partial z^{\prime}}\right](\mathrm{IX})+(\mathrm{V})(\mathrm{VIII}) .
\end{aligned}
$$

The passivity condition formed by taking the alternant of (14.9) with $\left(14.4_{2}\right)$ is found to be disappear identically in virtue of the other equations of the system. Hence (16.4), (16.7) represent all the integrability conditions of the differential system $(14.4,7,9)$; and clearly this system is not passive, since (16.4) certainly does not disappear identically, the coefficient of $\rho_{z}$ in this equation being $(\mathrm{I})^{2}$, which is $\neq 0$ by the hypothesis of Case IIb2.

Forming next the linear combination: (VII) times (16.4) minus (I) times (16.7), we eliminate the derivatives of $\rho$ and get

$$
(\mathrm{XIX}) \sigma_{z}+(\mathrm{XX}) \sigma_{z^{\prime}}=(\mathrm{XXI}) \rho+(\mathrm{XXII}) \sigma
$$

where

$$
(\mathrm{XIX})=(\mathrm{VII})(\mathrm{XI})-(\mathrm{I})(\mathrm{XV}),
$$

with similar expressions for (XX), (XXI), (XXII).

We now compare (16.9) with (16.2). First, suppose that these are independent as linear algebraic equations in $\sigma_{z}, \sigma_{z^{\prime}}, \rho, \sigma$, that is, that not all the two-rowed determinants in the following matrix vanish:

$$
\left\|\begin{array}{ccrr}
2(\mathrm{I}) & (\mathrm{X}) & -(\mathrm{VIII}) & -(\mathrm{IX}) \\
(\mathrm{XIX}) & (\mathrm{XX}) & (\mathrm{XXI}) & (\mathrm{XXII})
\end{array}\right\| .
$$


Then we consider two possibilities, namely, the determinant of the first two columns:

$$
2(\mathrm{I})(\mathrm{XX})-(\mathrm{X})(\mathrm{XIX})
$$

either does or does not vanish.

If it vanishes, we infer, by linear combination of (16.9) and (16.2), a relation of the form

$$
\cdot \rho+\cdot \sigma=0,
$$

where the dots represent known coefficients not both zero (for (16.9) and (16.2) are, by hypothesis, linearly independent).

If the coefficient of $\rho$ in (16.13) is zero, the coefficient of $\sigma$ cannot be zero; it follows that $\sigma=0$, contrary to the prescribed inequation $\sigma \neq 0$. There is then no solution of our differential system.

On the other hand, if the coefficient of $\rho$ in $(16.13)$ is $\neq 0$, we can solve this equation for $\rho$ in the form $\rho=\cdot \sigma$, and substituting in our original system $(14.4,7)$, we have the classical case of a linear differential system of first order in $\sigma$ alone-easily settled here by reference to the exactness or nonexactness of a certain readily constructed differential, $\cdot d x+\cdot d y+\cdot d z+\cdot d y^{\prime}$ $+\cdot d z^{\prime}$.

Suppose now that the determinant (16.12) does not vanish. Then (16.2), (16.9) are solvable in the form

$$
\sigma_{z}=\cdot \rho+\cdot \sigma, \quad \sigma_{z^{\prime}}=\cdot \rho+\cdot \sigma .
$$

Substituting this in the other equations of our system, (14.4), (14.7), (14.9), (16.4), we find a differential system in $\rho, \sigma$ of the form:

$$
\begin{array}{rlrl}
\frac{d \rho}{d x} & =\cdot \rho+\cdot \sigma, & \frac{d \sigma}{d x} & =\cdot \sigma, \\
\rho_{y} & =\cdot \rho_{z^{\prime}}+\cdot \rho+\cdot \sigma, & \sigma_{y} & =\cdot \rho+\cdot \sigma, \\
\rho_{z} & =\cdot \rho_{z^{\prime}}+\cdot \rho+\cdot \sigma, & \sigma_{z} & =\cdot \rho+\cdot \sigma, \\
\rho_{y^{\prime}} & =\cdot \rho_{z^{\prime}}+\cdot \rho+\cdot \sigma ; & \sigma_{y^{\prime}} & =\cdot \rho+\cdot \sigma, \\
& \sigma_{z^{\prime}} & =\cdot \rho+\cdot \sigma .
\end{array}
$$

All the passivity conditions which occur in the discussion of this system, as arrived at by formation of alternants of first order differential expressions, are evidently of the form

$$
\cdot \rho_{z^{\prime}}+\cdot \rho+\cdot \sigma=0 .
$$

Since there are at most three linearly independent equations of this type, we must arrive, after just a few additional steps, at a definite decision as to the solvability of our differential system and the generality of its solution. 
With this, we have disposed of the case of rank 2 of the matrix (16.11). Suppose, finally, that this matrix is of rank 1 (it cannot be of rank 0 , since $(\mathrm{I}) \neq 0$ ).

Then (16.7) is linearly dependent on (16.2) and (16.4), so that we have to adjoin only (16.4) as the sole passivity condition of the previously existent system. We now have in $(14.4,7,9)$ and $(16.4)$ a differential system which can be expressed in the following operational form:

$$
\begin{aligned}
\frac{d \rho}{d x} & =\cdot \rho+\cdot \sigma, \\
{\left[\frac{\partial}{\partial y^{\prime}}-\lambda \frac{\partial}{\partial z^{\prime}}\right] \rho } & =\sigma_{z^{\prime}}+\cdot \rho\left({ }^{31}\right), \\
{\left[\frac{\partial}{\partial y}-\lambda \frac{\partial}{\partial z}-(\mathrm{II}) \frac{\partial}{\partial z^{\prime}}\right] \rho } & =\cdot \sigma_{z^{\prime}}+\cdot \rho+\cdot \sigma, \\
{\left[2(\mathrm{I}) \frac{\partial}{\partial z}+(\mathrm{X}) \frac{\partial}{\partial z^{\prime}}\right] \rho } & =\cdot \sigma_{z^{\prime}}+\cdot \rho+\cdot \sigma ; \\
{\left[\frac{\partial}{\partial y^{\prime}}-\lambda \frac{\partial}{\partial z^{\prime}}\right] \sigma } & =\cdot \rho+\cdot \sigma, \\
{\left[\frac{\partial}{\partial y}-\lambda \frac{\partial}{\partial z}-(\mathrm{II}) \frac{\partial}{\partial z^{\prime}}\right] \sigma } & =\cdot \rho+\cdot \sigma, \\
{\left[2(\mathrm{I}) \frac{\partial}{\partial z}+(\mathrm{X}) \frac{\partial}{\partial z^{\prime}}\right] \sigma } & =\cdot \rho+\cdot \sigma .
\end{aligned}
$$

The important point to observe is that the operators on $\rho$ and on $\sigma$ are the same.

The fourth equation of (16.16) is the new one of this system, and the new passivity conditions are found by forming the alternant of this equation with the other ones of (16.16). The alternant with the first equation of (16.16) turns out to disappear identically in virtue of the other equations of the system.

The remaining alternants are of the first order in the partial derivatives of $\sigma$ as well as $\rho$, precisely because of the identity of corresponding operators in (16.16), (16.17). For instance, after forming the alternant of the second and fourth equations in (16.16), we have a term of the form

(31) Note that the coefficient of $\sigma_{z^{\prime}}$ in this equation is unity. The dots in the other equations represent, as usual, calculable known coefficients. 


$$
\left[\frac{\partial}{\partial y^{\prime}}-\lambda \frac{\partial}{\partial z^{\prime}}\right] \frac{\partial}{\partial z^{\prime}} \sigma,
$$

apparently of the second order; but this is equal to

$$
\left\{\frac{\partial}{\partial y^{\prime}}-\lambda \frac{\partial}{\partial z^{\prime}}, \frac{\partial}{\partial z^{\prime}}\right\} \sigma+\frac{\partial}{\partial z^{\prime}}\left[\frac{\partial}{\partial y^{\prime}}-\lambda \frac{\partial}{\partial z^{\prime}}\right] \sigma,
$$

where the braces denote the alternant of the two operators separated by the comma-and this alternant is of the first order. The second term is, by the second equation of (16.17), equal to

$$
\frac{\partial}{\partial z^{\prime}}(\cdot \rho+\cdot \sigma)
$$

a differential expression of first order. Hence the term (16.18) is equal to a first order expression in the partial derivatives of $\rho, \sigma$, and a similar reduction applies to other apparently second order terms arising in the formation of our passivity conditions.

In summary, we obtain two passivity conditions of the form

$$
\cdot \rho_{z^{\prime}}+\cdot \sigma_{z^{\prime}}+\cdot \rho+\cdot \sigma=0, \quad \cdot \rho_{z^{\prime}}+\cdot \sigma_{z^{\prime}}+\cdot \rho+\cdot \sigma=0 .
$$

If these two are linearly independent, then either they can be solved for $\rho_{z^{\prime}}, \sigma_{z^{\prime}}$ :

$$
\rho_{z^{\prime}}=\cdot \rho+\cdot \sigma, \quad \sigma_{z^{\prime}}=\cdot \rho+\cdot \sigma,
$$

or else a non-identically vanishing relation of the form $\cdot \rho+\cdot \sigma=0$ can be deduced from them by linear combination.

In the latter case, the discussion of our system is easily finished along lines already discussed (cf. (16.13)). In the former case, if we substitute (16.20) in (16.16), (16.17), we obtain a differential system in which every first partial derivative of $\rho, \sigma$ is expressed in the form $\cdot \rho+\cdot \sigma$. Then all the passivity conditions of this system are clearly of the form $\cdot \rho+\cdot \sigma=0$, so that the discussion of the system is easily concluded along the lines of standard theory $\left({ }^{32}\right)$.

If both equations (16.19) disappear identically, the system $(16.16,17)$ is completely in tegrable.

The only remaining case is where the matrix of the coefficients in (16.19) is of rank 1 , so that these equations amount to a single linearly independent one:

$$
\cdot \rho_{z^{\prime}}+\cdot \sigma_{z^{\prime}}+\cdot \rho+\cdot \sigma=0 .
$$

If the coefficient of $\rho_{z^{\prime}}$ is zero, then either we have $\cdot \rho+\cdot \sigma=0$, a case already

(32) Cf., as in connection with (13.15), Eisenhart, loc. cit. 
disposed of, or else $\sigma_{z^{\prime}}=\cdot \rho+\cdot \sigma$. In the latter case, we obtain by substitution in $(16.16,17)$ a system of the form $(16.15)$, already discussed.

Accordingly, we may suppose (16.21) solved for $\rho_{z^{\prime}}$ :

$$
\rho_{z^{\prime}}=\cdot \sigma_{z^{\prime}}+\cdot \rho+\cdot \sigma .
$$

Let us form the alternant of this with the second equation of (16.16):

$$
\frac{\partial}{\partial z^{\prime}}\left(16.16_{2}\right)-\left[\frac{\partial}{\partial y^{\prime}}-\lambda \frac{\partial}{\partial z^{\prime}}\right](16.22) \text {. }
$$

Then we get a passivity condition of the form

$$
\sigma_{z^{\prime} z^{\prime}}=\cdot \sigma_{z^{\prime}}+\cdot \rho+\cdot \sigma,
$$

solvable for $\sigma_{z^{\prime} z^{\prime}}$, whose coefficient in (16.23) is evidently 1. There are two additional passivity conditions, obtained by building the alternant of (16.22) with the third and fourth equations of $(16.16)\left({ }^{33}\right)$; these are of the form

$$
\cdot \sigma_{z^{\prime} z^{\prime}}+\cdot \sigma_{z^{\prime}}+\cdot \rho+\cdot \sigma=0 \text {. }
$$

Substituting in this the expression (16.24) for $\sigma_{z^{\prime} z^{\prime}}$, we get two equations of the type

$$
\cdot \sigma_{z^{\prime}}+\cdot \rho+\cdot \sigma=0, \quad \cdot \sigma_{z^{\prime}}+\cdot \rho+\cdot \sigma=0 .
$$

Unless both of these disappear identically, we can deduce either $\cdot \rho+\cdot \sigma=0$, $\sigma_{z^{\prime}}=\cdot \rho+\cdot \sigma$. In either case we find ourselves in situations already discussed $\left({ }^{34}\right)$, and our investigation finishes along simple lines of standard theory.

If, however, both equations (16.25) disappear identically, we have to adjoin (16.22), (16.24) as the passivity conditions of the system $(16.16,17)$. Then, clearly, the extended system has only the three parametric derivatives $\sigma_{z^{\prime}}, \rho, \sigma$; consequently all further passivity conditions must be of the form

$$
\cdot \sigma_{z^{\prime}}+\cdot \rho+\cdot \sigma=0 \text {. }
$$

Evidently, our investigation now comes to its conclusion by reduction to cases already considered.

We may conclude by observing that throughout the discussion of Case $\mathrm{IIb}$, even in the most unfavorable eventualities, the only derivative of an order higher than the first which can occur is $\sigma_{z^{\prime} z^{\prime}}$.

17. Case III. This is the case of rank two of the matrix $\Delta$, that is:

$$
\left|\begin{array}{lll}
A & B & C \\
A_{1} & B_{1} & C_{1} \\
A_{2} & B_{2} & C_{2}
\end{array}\right|=0, \quad\left\|\begin{array}{ccc}
A & B & C \\
A_{1} & B_{1} & C_{1}
\end{array}\right\| \neq 0 .
$$

(33) The condition obtained by alternating with $d / d x$ (first equation of (16.16)) disappears in virtue of the other equations in our system.

(34) As is readily seen in the latter case by combining $(16.26),(16.22)$ with $(16.16,17)$. 
Let us denote the determinants in the latter matrix as follows:

$$
\Delta_{1} \equiv B C_{1}-C B_{1}, \quad \Delta_{2} \equiv C A_{1}-A C_{1}, \quad \Delta_{3} \equiv A B_{1}-B A_{1} ;
$$

then according to (17.1), at least one of $\Delta_{1}, \Delta_{2}, \Delta_{3}$ is not zero.

It follows, by the linear equations $A L+B M+C N=0, A_{1} L+B_{1} M+C_{1} N$ $=0$, of (7.5), (7.7), that a proportionality factor $\rho$, function of $x, y, z, y^{\prime}, z^{\prime}$, exists such that

$$
L=\rho \Delta_{1}, \quad M=\rho \Delta_{2}, \quad N=\rho \Delta_{3} .
$$

$\rho$ is now the sole unknown function of our problem.

By (17.3),

$$
L N-M^{2}=\rho^{2}\left(\Delta_{1} \Delta_{3}-\Delta_{2}^{2}\right) ;
$$

hence, by the fundamental inequation $L N-M^{2} \neq 0$ of (7.5), we must have

$$
D \equiv \Delta_{1} \Delta_{3}-\Delta_{2}^{2} \neq 0
$$

-otherwise, we can conclude immediately that the given curve family is nonextremal.

We are thus led to make the following subdivision of Case III :

Case IIIa. $\quad D \neq 0$,

Case IIIb. $\quad D=0\left({ }^{35}\right)$.

Example of Case IIIb.

$$
y^{\prime \prime}=y^{2}+z^{2}, \quad z^{\prime \prime}=0 .
$$

We calculate by (7.6), (7.9):

$$
\begin{aligned}
& A=-4 z, \quad B=4 y, \\
& C=0 \text {; } \\
& A_{1}=-4 z^{\prime}, \quad B_{1}=4 y^{\prime}, \\
& C_{1}=0 \text {; } \\
& A_{2}=0 \text {, } \\
& B_{2}=4\left(y^{2}+z^{2}\right), \\
& C_{2}=0 \text {. }
\end{aligned}
$$

Here

$$
\Delta_{1}=0, \quad \Delta_{2}=0, \quad \Delta_{3}=16\left(y z^{\prime}-z y^{\prime}\right) ;
$$

therefore $D=0$.

The curve family (17.8) is nonextremal.

Cáse IIIa. Let us substitute (17.3) for $L, M, N$ in the differential system

(35) Geometrically expressed, (17.3), where $\rho$ is regarded as a parameter, represents a line in $L M N$-space passing through the vertex of the critical cone. The distinction between Cases IIIb, and IIIa, respectively, consists in whether this line is or is not an element of the critical cone. 
(7.5) as well as in the equations (8.8), (8.9) of its extended system. Then we obtain exactly five independent linear equations in the five first order derivatives of $\rho$; and these equations can be solved for the derivatives in question, giving

$$
\rho_{x}=E_{1} \rho, \quad \rho_{y}=E_{2} \rho, \quad \rho_{3}=E_{3} \rho, \quad \rho_{y^{\prime}}=E_{4} \rho, \quad \rho_{z^{\prime}}=E_{5} \rho .
$$

Here the coefficients $E_{i}$ are calculable known functions, being rational expressions in the partial derivatives of $F, G$ each having $D$ for denominator; accordingly, the $E_{i}$ exist as determinate quantities in virtue of the stipulation $D \neq 0$.

By (17.4), (17.5), the fundamental inequation $L N=M^{2} \neq 0$ is seen to be expressed precisely by requiring a solution $\rho$ of the system (17.10) other than the trivial one, zero:

$$
\rho \neq 0 \text {. }
$$

For the existence of such a $\rho$, the exactness of the differential

$$
E_{1} d x+E_{2} d y+E_{3} d z+E_{4} d y^{\prime}+E_{5} d z^{\prime}
$$

is evidently a necessary and sufficient condition. If, in case of exactness, the integral of this differential is $\zeta\left(x, y, z, y^{\prime}, z^{\prime}\right)$, then

$$
\rho=e^{\zeta} \text {. }
$$

$\zeta$ is determined up to an additive constant, and $\rho$, therefore, up to a constant factor.

After $\rho$ has been found, we substitute in (17.3) to obtain $L, M, N$, and therefrom we find $\phi$ by the method of $\S 5 . L, M, N$ will be determined up to a constant factor, since that is the case with $\rho$. By the relations (7.3) and by $\S 5$, the integrand $\phi$ is therefore determined up to a constant factor, besides the usual additive total derivative as to $x$-thus, according to the convention associated with (3.7), $\phi$ is essentially uniquely determined.

The results of this section are expressed in Theorems VI and VII of $\S 3$.

Example of Case IIIa.

$$
y^{\prime \prime}=z^{2}, \quad z^{\prime \prime}=y^{2} .
$$

By calculation, using (7.6), (7.9):

$$
\begin{aligned}
A=-4 z, & B=0, & C=4 y ; \\
A_{1}=-4 z^{\prime}, & B_{1}=0, & C_{1}=4 y^{\prime} ; \\
A_{2}=-4 y^{2}, & B_{2}=0, & C_{2}=4 z^{2} .
\end{aligned}
$$

The conditions (17.1) and (17.5) are immediately verifiable.

Since $\Delta_{1}=0, \Delta_{3}=0$, equations (17.3) give $L=0, N=0$.

By substitution in the differential system (7.5), (8.8), (8.9), this becomes 
the following system for $M$ (which, in this example, plays the part of $\rho$ ):

$$
M_{x}=0, \quad M_{y}=0, \quad M_{z}=0, \quad M_{y^{\prime}}=0, \quad M_{z^{\prime}}=0 .
$$

Therefore $M=$ const., and since a constant factor is inessential, we may say $M=1$. Knowing now $L, M, N$, we can easily find $\phi$ by the method of $\S 5$; the result is

$$
\phi=y^{\prime} z^{\prime}+\frac{1}{3} y^{3}+\frac{1}{3} z^{3},
$$

and this is essentially unique, that is, within the slight possibility of modification expressed by (3.7).

18. Case IV. This is the case where the determinant of the fundamental matrix $\Delta$ does not vanish :

$$
\left|\begin{array}{lll}
A & B & C \\
A_{1} & B_{1} & C_{1} \\
A_{2} & B_{2} & C_{2}
\end{array}\right| \neq 0
$$

that is, $\Delta$ is of rank three.

Since (18.1) contradicts the necessary condition (7.10) for an extremal family, any curve family coming under Case IV is certainly nonextremal.

Example.

$$
y^{\prime \prime}=y^{2}+z^{2}, \quad z^{\prime \prime}=y .
$$

By calculation, with reference to (7.6), (7.9):

$$
\begin{aligned}
& A=-4 z, \quad B=4 y, \quad C=2 ; \\
& A_{1}=-4 z^{\prime}, \quad B_{1}=4 y^{\prime}, \quad C_{1}=0 \text {; } \\
& A_{2}=-4 y, \quad B_{2}=4\left(y^{2}+z^{2}\right), \quad C_{2}=0 .
\end{aligned}
$$

The determinant of the matrix $\Delta$ is not equal to zero. The curve family (18.2) is therefore non-extremal.

\section{Columbia University, NEW YoRK, N. Y.}

